Meta

Journal des tradlucteurs

Translators' Journal

\title{
Les grandes banques de terminologie
}

\section{René Gagnon}

Volume 39, numéro 3, septembre 1994

URI : https://id.erudit.org/iderudit/002783ar

DOI : https://doi.org/10.7202/002783ar

Aller au sommaire du numéro

Éditeur(s)

Les Presses de l'Université de Montréal

ISSN

0026-0452 (imprimé)

1492-1421 (numérique)

Découvrir la revue

Citer cette note

Gagnon, R. (1994). Les grandes banques de terminologie. Meta, 39(3), 498-520. https://doi.org/10.7202/002783ar d'utilisation que vous pouvez consulter en ligne.

https://apropos.erudit.org/fr/usagers/politique-dutilisation/ 


\section{LES GRANDES BANQUES DE TERMINOLOGIE}

\section{AVANT-PROPOS}

Les informations rapportées dans l'article suivant ne sont pas toujours de la dernière actualité, une partie des sources citées remontant aux années 1960 et 1970 , les plus récentes franchissant à peine le seuil des années 1990. De plus, les données relatives aux banques de terminologie canadiennes sont beaucoup plus abondantes que celles qui concernent les banques européennes.

La première observation au sujet de l'actualité des informations s'explique en partie par le fait que le texte a été rédigé en 1990-1991, après que la documentation nécessaire eut été rassemblée. Il ne sert d'ailleurs pas tant à exposer un état de la situation le plus actuel possible ni à dévoiler la fine pointe en matière d'évolution des banques de terminologie qu'à détecter les causes du succès des banques de terminologie en général, des plus importantes d'entre elles en particulier. Il a donc fallu étudier leur fonctionnement au long des décennies, l'évolution de leur organisation, leur croissance, leur histoire et leur accession au statut de «grande banque». Dans cette perspective, les aspects historiques de la recherche ont eu priorité sur l'exclusivité des dernières nouvelles: eux seuls permettaient d'identifier les conditions favorisant l'émergence et le développement des banques de terminologie

Quant à la deuxième observation relevant l'écart qui sépare la quantité d'informations sur les banques canadiennes et européennes, voici comment elle se justifie: j'ai une connaissance pratique des banques canadiennes puisque je les ai utilisées fréquemment. Par contre, à part quelques démonstrations données lors d'occasions spéciales, les banques européennes se sont fait connaître au Québec uniquement par des articles de périodiques et des extraits d'ouvrages spécialisés. Or ces références ont ici une disponibilité réduite. En outre, la plupart des articles traitant des banques allemandes ou scandinaves sont écrits en allemand, en suédois, ou autre; après qu'on les ait choisis, après qu'on les ait traduits, quelques-uns trouvent la voie de la publication en français ou en anglais. Ils offrent alors une information déphaséc par rapport à l'actualité et quelquefois même par rapport à la réalité. Qu'on imagine un peu la difficulté de celui qui, sans avoir l'usage du français, aurait à rédiger un article sur la BTQ ou sur FRANTERM..

\section{INTRODUCTION}

L'exposé suivant s'appuie sur une définition de la notion banque de terminologie élaborée après la compilation d'un dossier terminologique et l'étude d'une douzaine d'autres définitions. Elle se formule comme suit :

Système informatique de gestion de données destiné au stockage, au traitement et à la diffusion d'informations terminologiques ou d'intérêt terminologique.

Objectivement, les banques décrites ici appartiennent toutes à la classe des grandes banques de terminologie. Cette qualité leur est conférée par la reconnaissance des spécialistes du domaine qui n'ont pu ignorer leur succès ni les dimensions extraordinaires qu'elles avaient prises. L'utilisation de l'expression «grande banque» ne devrait pas choquer: elle résulte de l'application stricte d'un ensemble de critères et ne vise pas à glorifier certaines banques au détriment des autres; elle ne comporte aucun jugement de valeur. Elle dresse le constant de leur taille, de leur dimension. On aboutit d'ailleurs aux mêmes conclusions en appliquant le principe de la sélection naturelle (aussi contestable soit-il) : aux différentes étapes de leur conception, de leur constitution et de leur croissance, les grandes banques ont dû trouver des solutions originales à de nombreux problèmes inédits, leur aptitude à surmonter les épreuves confirmant leur viabilité. Aux objectifs que leurs concepteurs avaient arrêtés sont venus s'ajouter des impératifs de rentabilité (en termes financiers ou de recherche et développement), la justification politique de tels investissements à fonds perdu, sans compter les contingences de l'utilisation.

\section{IDENTIFICATION DES \\ GRANDES BANQUES}

L'expression grande banque de terminologie n'est pas originale ni nouvelle. Dès 1979, Sager et Johnson parlent de large term banks. Dans leur article (p. 2), ils identifient EURODICAU-TOM, LEXIS, TEAM, la BTUM, la BTQ et la banque du Institut für Angewandte Sprachwissenschaft und Rechenzentrum de la Technische Universität Dresden. Un an plus 
tard, Sager et McNaught (1980:33) emploient l'expression large organisations au sujet d'EURODICAUTOM, LEXIS et TEAM. Puis quand Hvalkof (1982: 5) publie une étude fouillée sur la faisabilité d'échanger des données terminologiques d'une banque à l'autre, elle fonde ses comparaisons sur la BTQ, EURODICAUTOM, NORMATERM, LEXIS et TEAM. Vers la même époque, Hotz (1983: 64 et ss.) reconnaît l'expression puisqu'il l'emploie à plusieurs reprises; il décrit alors cinq banques (BTQ, EURODICAUTOM, LEXIS, TEAM, TERMIUM) qu'il classe parmi les grandes banques, en mentionnant, prudemment, qu'il en existe d'autres appartenant à la même catégorie. De Bessé (1983: 161) parle aussi de grande banque et cite les mêmes que Hotz. En 1983, à Hull, se tient la première réunion internationale des gestionnaires de grandes banques (Cardin 1983:8) à laquelle participent pleinement les représentants de la BTQ, d'EURODICAUTOM, de LEXIS, de TEAM et de TERMIUM, tandis que ceux de NORMATERM et de quelques autres y tiennent le rôle d'observateurs. Au terme de la réunion, seuls les représentants des cinq institutions mentionnées signent l'accord auquel on en arrive, et dont l'article 1 (Cardin 1983:8) se lit comme suit :

1. Les banques BTQ, EURODICAUTOM, LEXIS, TEAM et TERMIUM conviennent qu'elles utiliseront le format MATER [...].

Par la suite, la reconnaissance d'une classe composée de grandes banques semble chose acquise auprès des spécialistes qui auront à aborder la question: Felber (1984: 352) parle de big terminological data banks, Baudot (1986: 155-156) de grande banque de terminologie, Abbou (1987: 214) de grandes bases de données internationales terminologiques, L'Homme (1988: 32) de grandes banques de terminologie ou de banques de terminologie importantes, Schneider (1987: 210) de large data banks ou large banks, Wells (1988: 58) de grande banque de terminologie, et ainsi de suite. À remarquer que la plupart n'identifient pas celles qu'ils classent parmi les grandes banques. Ils ne situent pas non plus les limites séparant les grandes banques des autres. Aussi, le moyen d'éviter l'arbitraire dans l'attribution de l'étiquette grande banque consisterait à énoncer des critères comme autant de conditions essentielles à l'inclusion dans la classe des grandes banques.

Bien que plusieurs spécialistes aient abordé la question plus ou moins directement, il ne s'en trouve pas qui énumère clairement, délibérément, les critères en question. L'Homme (1988: 31) résume bien, dans un seul paragraphe, ce qu'on trouve éparpillé chez d'autres auteurs:

Les banques de terminologie importantes sont celles qui ont été mises au point pour ou par des organismes gouvernementaux ou des grandes sociêtés. Les coûts de l'élaboration de telles banques sont très élevés. Le nombre de termes traités dépasse souvent le million, ce qui néces- site une grande capacité de traitement, de mémoire et une mise à jour intensive.

L'analyse de cet extrait permet d'identifier quatre critères :

1) ressources financières importantes;

2) nombre de termes traites ;

3) ressources informatiques considérables;

4) ressources humaines considérables.

Si les trois premiers semblent évidents, le quatrième est tributaire de la grande capacité de traitement, de mémoire et une mise à jour intensive, dans le sens où le fonctionnement de toute grande banque doit procéder d'un noyau dur de langagiers s'appuyant sur un réseau plus ou moins imposant d'informaticiens et de spécialistes en tous genres.

Les critères 1 (ressources financières), 3 (ressources informatiques) et 4 (ressources humaines) font tous reférence à une structure administrative complexe et aux moyens considérables qu'exige l'accession au statut de grande banque. Aucune équivoque possible sur ce point : on sait qu'un chercheur isolé ou qu'une équipe très réduite disposant de moyens limités et d'une organisation déficiente ne parviendra pas à mettre sur pied ce qui pourrait éventuellement devenir une grande banque. Quant au nombre de termes, il s'agit là encore d'un critère facile à dégager et à appliquer.

Il reste au moins deux autres critères qui devraient s'ajouter aux quatre précédents :

5) durée ;

6) réseau de diffusion.

La durée, comme condition d'accession au statut de grande banque, est justifiée par la nature même de tels projets qui exigent de longues années de planification et de mise en place. Ils doivent s'inscrire dans le temps et marquer leur époque. Voilà toutefois qui soulève une question épineuse : comment déterminer la période de gestation d'une grande banque ? Y-a-t-il un nombre minimal d'années pour parvenir à ce statut ? Peut-on appliquer ce critère de la durée isolément (comme celui du nombre de termes), ou bien est-il trop étroitement lié aux autres critères considérés, entre autres aux moyens techniques et financiers investis dans la mise sur pied du système? Autrement dit, est-il possible d'identifier, après une durée donnee, le moment où une banque de terminologie devient un outil au plein sens du terme? Bien malin ou bien téméraire celui qui oserait avancer des réponses définitives à ces questions.

Et il en va du dernier critère, celui du réseau de diffusion, comme du cinquième : comment définir un réseau de diffusion terminologique satisfaisant? Combien d'utilisateurs doit-il avoir, en accès direct ou différé, pour qu'on parle de réseau? Faut-il établir une frontière entre des banques expérimentales et celles qui sont devenues un outil et qui, au moyen d'un réseau d'utilisateurs directs ou indirects, ont pu 
contribuer à la diffusion des terminologies qu'on y avait stockées? Comment mesurer l'impact d'une banque, son influence, sa fécondité en matière de diffusion de terminologies auprès des clientèles cibles? Comment départager ce qui provient de son influence de ce qu'on peut attribuer à la compétence et à l'expérience des langagiers concernés ?

Une évaluation plus poussée des implications d'une grille d'évaluation ainsi conçue nous entraîne vers d'autres réflexions quant à ses limites: l'arbitraire réapparaît forcément quand il est question, pour chaque critère, de déterminer un seuil au-delà duquel une banque de terminologie passe au rang de grande banque. L'application de la grille soulève aussi des problèmes d'un autre ordre: que faire par exemple quand une banque satisfait à quatre ou cinq critères, mais pas aux six? Y a-t-il une hiérarchie des critères telle qu'il suffirait de remplir les conditions posees par un certain nombre d'entre eux (les cinq premiers, par exemple) pour qu'une banque soit considérée comme grande? Autrement dit, s'agit-il, dans tous les cas, de critères universels et absolus?

Enfin, aussi objectif qu'on souhaite l'être, il convient de préciser que les données dont on dispose risquent à l'occasion d'être incomplètes, périmées ou, pis encore, fausses. Un tel état de fait s'explique de diverses manières: d'abord, l'accès à l'information n'est pas aussi facile pour toutes les banques. Par ailleurs, cet accès passe le plus souvent par des canaux officiels où on ne révèle que ce qui vaut d'être révélé, en conformité avec des objectifs bien différents des nôtres. De plus, la dernière édition du répertoire le plus complet, celui de Krommer-Benz, remonte à 1985. Et ce n'est pas en complétant les données qu'on $\mathrm{y}$ trouve avec des informations parcellaires comme celles publiées dans TermNet News, seule source d'information sur le sujet, et malgré tout son sérieux et sa fiabilité, qu'on parviendra à une information complète, à jour et fiable.

Aussi, à cause de l'imprécision des critères euxmêmes, à cause de la difficulté que représente leur application et de la plus ou moins grande fiabilité des informations dont on dispose, on en est réduit à douter des vertus mêmes d'un tableau comparatif élaboré sur leur base. Et puis un survol des données disponibles, réparties ou non sur un tel tableau, convainc sans peine du bien-fondé de la position préconisée par les organisateurs de la réunion de 1983 à Hull, de même que de la pertinence des choix faits par Hotz (1983), Schneider (1987: 208) et Wells (1988: 58) : cinq banques forment une classe à part où elles se distinguent aux points de vue de leur maturité, de leur dimension, de leurs actions et de leur diffusion. Il s'agit de la BTQ, d'EURODICAUTOM de LEXIS, de TEAM et de TERMIUM. Depuis 1983 , de nouvelles banques sont apparues, certaines on disparu, tandis que la plupart ont continué de croître. D'autres banques peuvent certes être considérées comme grandes et leur réussite mérite d'être signalée : DANTERM, TERMCAT, le réseau des banques scandinaves (NORDTERM), NORMATERM, CÉZEAU-
TERM, etc. Mais elles n'appartiennent pas à la même classe et ne satisfont pas aux six critères énumérés plus haut.

\section{LA BANQUE EUROPÉENNE : EURODICAUTOM}

Situation

La banque de la Commission des Communautés européennes est bicéphale : basée à la fois au Luxembourg et à Bruxelles, elle voit ses activités, ses effectifs, ses utilisateurs, sa direction et sa diffusion répartis dans deux capitales. EURODICAUTOM est gérée par les Bureaux de terminologie de la Commission des Communautés européennes (CCE) à Luxembourg et à Bruxelles (Goetschalckx 1981a: 76). Ainsi, par exemple, les équipes de terminologues ont leurs bureaux dans les deux capitales, et si l'ordinateur (Siemens 7760) est situé à Luxembourg, la masse critique du réseau de diffusion se trouve par contre à Bruxelles, là où siègent la $\mathrm{CCE}$, le Parlement européen, l'OTAN, de nombreuses institutions d'enseignement de la traduction (universités et instituts), etc.

La conception du DICAUTOM (pour dictionnaire automatique), l'ancêtre d'EURODICAUTOM, fut inspirée des expériences de traduction automatique menées aux États-Unis à partir du début des années 1950 , en ceci qu'il s'agissait d'un auxiliaire à la traduction visant à améliorer le rendement des traducteurs. Bachrach (1987: 97) fait observer que le dictionnaire électronique était en premier lieu destiné à servir d'outil de travail au traducteur humain et que, à ce titre, il devait réduire la trop grande portion de temps $(50 \%)$ que le traducteur consacrait à la recherche terminologique (Bachrach 1971: 106). Sa mise sur pied remonte à 1963 quand J. Albert Bachrach, du Bureau de terminologie de la Communauté européenne du charbon et de l'acier (CECA), à Luxembourg, entreprend les travaux en compagnie de Lydia Hirschberg de l'Université libre de Bruxelles (Bachrach $1981: 27$; Hirschberg $1965: 78$ ).

On prend vite conscience toutefois que l'accès aux données du DICAUTOM est limité par deux facteurs : 1) l'insuffisance de matériel et de personnel pour effectuer les recherches en différé et pour traiter les demandes, et 2) l'insuffisance du contenu. Comme la Haute Autorité de la CECA est dissoute simultanément à la création de la Communauté économique européenne (CEE) en 1965, les investissements et les travaux s'interrompent dès lors (Bachrach $1987: 99$ ). En parallèle, M. C. Cuypers, attaché au Bureau de terminologie du Marché commun, mettait sur pied EUROTERM, un système de terminologie automatique (Goetschalckx 1981b: 31). En 1969, à la suite d'une décision administrative de la Commission à Luxembourg, les systèmes existants sont fusionnés et vont désormais porter le nom d'EURODICAUTOM, sans pour autant que les travaux ne reprennent quelque vigueur: c'est en 1973 seulement que le chantier pourra redémarrer (Reichling $1981: 40$ ). 
Les visées initiales des concepteurs du DICAUTOM étaient plutôt limitées. Hirschberg (p. 78) déclarait en 1965 :

Le cadre pragmatique dans lequel nous nous trouvons est celui de la construction de dictionnaires automatiques de locutions pour traducteurs humains, français-néerlandais et néerlandaisfrançais $[\ldots]$

Mais, avec les années, avec l'évolution économique, sociale et politique de l'Europe, les objectifs et les préoccupations du début prirent de l'ampleur. Comme suite à la formation d'EURODICAUTOM et à son intégration aux activités langagières de la CCE comme conséquence aussi de l'élargissement progressif de la Communauté européenne, on est passé depuis 1987 à un total de neuf langues à traiter: français, anglais, allemand, italien, néerlandais, danois, espagnol, portugais et grec. La complexité des travaux terminologiques s'est accrue dans la même mesure : comme bon nombre de textes issus de la CCE fon jurisprudence à l'intérieur de chacun des États membres, la centralisation et la standardisation des terminologies effectuées par EURODICAUTOM en sont devenues d'autant plus précieuses. C'est ainsi qu'au but premier d'améliorer la productivité et la qualité de la traduction faite pour le compte de la Commission et des institutions intégrées au réseau est venu s'ajouter l'objectif d'uniformiser la terminologie propre aux sujets d'intérêt commun tant à l'intérieur de la CCE que dans les pays membres.

\section{Ressources terminologiques}

Le DICAUTOM était destiné à l'enregistrement de locutions, dites unités phraséologiques, où les mots se retrouvent en contexte. La première version d'EURODICAUTOM poursuivait dans la même veine: en 1975 , la banque contenait 80 \% d'unités phraséologiques. Mais là aussi, les choses allaient évoluer; on y trouve maintenant diverses catégories de données : deux types d'unités phraséologiques, celles composées d'une phrase complète, et d'autres comportant seulement un membre de phrase sans qu'il s'agisse à proprement parler d'un syntagme, des entrées terminologiques (unitermes ou multitermes) accompagnées de définitions ou de contextes et d'autres, uniquement lexicales (Goetschalckx 1981a : 77). Ces informations sont consignées sur un support qu'on appelle entité et qui regroupe les données traductionnelles et terminologiques qu'on a pu rassembler autour d'une notion Le contenu d'EURODICAUTOM est réparti dans deux bases de données, l'une, le fichier-maître ou fichier-texte, réservée aux entités terminologiques, et l'autre, créée en 1980, répertoriant les abréviations (Hotz 1983 : 49; Reichling 1981: 54). Début 1987, DIANEGUIDE y dénombrait 380000 entités terminologiques et plus de 90000 abréviations (p. 51). Une dizaine de mois plus tard, Jacques Goetschalckx, à l'occasion du Congrès international sur la terminologie et le génie de la connaissance à Trèves, établissait quant à lui le contenu de la banque à 450000 entités terminologiques et à 120000 abréviations (Quicheron 1988: 149). Plus de $95 \%$ de ces entités contiennent du français et / ou de l'anglais et, à cause des efforts d'intégration des nouveaux membres de la CEE, on comptait déjà à cette époque 175000 entités contenant de l'espagnol, 110000 contenant du portugais et 70000 pour le grec (Quicheron $1988: 149$ ). Chaque entité peut avoir un contenu varié en termes de langues et de densité documentaire : la plupart ne comportent pas neuf langues; à l'occasion, on n'y trouve ni définition, ni contexte. Aussi certains preferent-ils calculer le contenu d'EURODICAUTOM par paires de termes : Krommer-Benz (p. 56) en comptait ainsi 1,7 million en 1985.

Initialement, les concepteurs du DICAUTOM rejettent l'idée de verser intégralement des dictionnaires et des glossaires existants dans leur banque. Ils préfèrent stocker les informations tirées de textes originaux dans chacune des langues de travail de la Communauté (Bachrach 1971 : 106-107). Mais quand, dans le milieu des années 1970, l'orientation est prise de faciliter une utilisation massive de la banque et qu'on réévalue de manière idoine les priorités de stockage, on parvient à la conclusion qu'il faut consigner des termes aussi bien que des unités phraséologiques. On en viendra aussi progressivement à profiter des modalités d'échange d'information avec d'autres banques (NORMATERM et TERMIUM notamment). Et puis les langagiers des institutions communautaires seront mis à contribution de manière beaucoup plus intensive (Bachrach 1987: 101; Hotz 1983 : 49). Pour comprendre comment s'effectue cette intégration des efforts, des énergies et des ressources des langagiers communautaires au réseau d'EURODICAUTOM, il suffit de lire un article de J. R. Mengarduque (1989: 155 et ss.) du Service de traduction du ministère néerlandais des Affaires étrangères, qui explique le fonctionnement de la filière d'alimentation de la banque : si les traducteurs et terminologues consultent la banque, ils doivent démontrer en contrepartie un grand intérêt à signaler tout nouveau besoin, à transmettre toute nouvelle information, à corriger les entités erronées et à compléter les incomplètes.

Tant l'acquisition que la production ou la préparation de nouvelles terminologies sont soumises aux prévisions des Bureaux de terminologie à qui on signale quels sont les projets de traduction les plus importants et les plus volumineux, à qui on soumet aussi des listes de secteurs lacunaires en terminologie, à qui, en somme, on mentionne ses besoins (Hotz 1983 : 55-56). En 1983-1984, le stockage de nouvelles entités se faisait au rythme de 22000 par année, en six langues (Rondeau 1984: 163). Mais cette cadence s'était considérablement accrue quelques années plus tard, du moins si l'on se reporte aux chiffres donnés, d'une part, dans DIANEGUIDE fin 1986 et ceux fournis par Goetschalckx en octobre 1987: là, on serait passé à un taux d'accroissement d'environ 80000 entités par année, sans égard au nombre de langues impliquées. Quoi qu'il en soit, le stockage 
massif d'informations en provenance de sources si diverses ne peut aller sans créer quelques problèmes de redondance (Reichling $1981: 51-52$ ).

Dès sa conception achevée en 1972, la Direction générale traduction de la CCE implantait la classification Lenoch ${ }^{1}$ tant pour ses fins de classement terminologique que documentaire. Il s'agit en fait d'un système de gestion des connaissances (Wellenstein 1989 : 107) plutôt que d'un thésaurus, conçu en vue de la consultation par des profanes. Inspiré de la classification décimale universelle (Reichling 1981: 81), il comporte quarante-cinq descripteurs principaux, en français, désignés par deux lettres, en plus d'un certain nombre de sous-rubriques (ou sous-descripteurs) représentés par un chiffre (Berteloot, Mulders et Winterhoff 1988: 142; Wellenstein 1989: 108). Pour permettre son classement, d'une part, et pour faciliter l'accès aux données qu'elle contient, d'autre part, chaque entité terminologique comporte un (ou quelques-uns) de ces codes. Amendé une première fois en 1975 alors qu'on y ajouta des annexes, le système a traversé les années inchangé jusqu'à tout récemment : on s'emploie maintenant à en corriger les défauts et à le mettre à jour (Wellenstein 1989: 107).

\section{Exploitation}

Personnel : Parce que ce genre de données varie beaucoup d'une année à l'autre et d'un auteur à l'autre, il est impossible d'établir une fois pour toutes quelles sont les ressources humaines dont dispose EURODICAUTOM pour son alimentation, son entretien et sa mise en jour, d'autant que les utilisateurs communautaires ont souvent la possibilitế, voire la responsabilité morale, de contribuer à son alimentation et à sa mise à jour. En 1985, Krommer-Benz (pp. 54-56) évaluait à près de soixante-quinze employés le personnel des deux Bureaux de terminologie de la CCE (trente-quatre à Bruxelles et trente-neuf à Luxembourg), dont plus d'une quarantaine de terminologues, sept documentalistes / bibliothécaires et environ vingtcinq autres professionnels, surtout des informaticiens. Il convient cependant de préciser que tout ce personnel n'est pas affecté exclusivement à EURODICAUTOM : en ce qui concerne les terminologues, en particulier, leur tâche principale consiste à appuyer le travail des traducteurs. Il demeure par ailleurs extrêmement difficile de quantifier la contribution des terminologues et traducteurs du réseau de diffusion, en particulier de ceux qui appartiennent à des organismes communautaires.

Matériel : Après avoir employé un logiciel maison tout au cours de l'histoire du DICAUTOM, la banque a été montée sur STAIRS ${ }^{2}$ en 1975 (Goetschalckx 1981b: 33), ce qui semblait logique puisqu'on employait alors un ordinateur IBM. En 1977, on passe à du matériel Siemens tant en ce qui concerne les ordinateurs que les périphériques (Hotz 1983 : 48). Puis en 1981-1982, on procède à l'implantation du logiciel de conception domestique Eurodicautom 2 aux fins du stockage, du classement et de l'interrogation de la banque (Krommer-Benz 1985 : 55).
Produits : Le DICAUTOM tirait son nom du fait que la recherche dans les mémoires de l'ordinateur se faisait de manière automatique sans pour autant qu'il s'agisse d'une recherche élémentaire. Au contraire. Le repérage des unités simples ou complexes s'y faisait selon des paramètres et des procédures complexes (Reichling 1981 : 44) en suivant le principe du largest match ou réponse partielle (c'est-à-dire que le syntagme est décomposé automatiquement en ses composantes) lorsqu'une réponse complète ne pouvait être offerte à l'utilisateur. À cette époque, les questions étaient constituées d'unités phraséologiques ou au moins de multitermes (Goetschalckx 1981a: 77 et 1981b: 31). On comprend mieux le procédé si on garde à l'esprit le fait que le DICAUTOM contenait surtout des unités phraséologiques, sinon des phrases complètes, et que l'interrogation s'y faisait par mots clés introduits sur cartes perforées; la réponse prenait la forme d'une liste imprimée des contextes les plus semblables possible dans la langue de départ, accompagnés de leur traduction dans la langue d'arrivée (Bachrach 1981: 27-28). Cette méthode reposait sur deux prémisses: 1) que l'utilisateur final exécute la consultation directement; et 2 ) qu'il exerce ses facultés critiques (Goetschalckx 1981b : 31).

Comme cette consultation automatique implique la reconnaissance du mot dans ses formes non canoniques (fléchies ou accordées), elle autorise la troncation postérieure dans l'interrogation. L'evolution matérielle et logicielle du système n'a pas remis en cause ces principes de départ, mais quand la base du stockage a cessé d'être l'unité phraséologique et que le nombre de langues consignées dans la banque a augmentê, on est passé à un mode d'interrogation où la consultation repose plutôt sur les termes d'une langue source vers une ou plusieurs langues cibles. La réponse peut, à la demande, ne contenir qu'une correspondance terme à terme, une phrase, un segment plus ou moins analogue correspondant vaguement aux termes de la question, etc. (Sager et Mc Naught 1980: 14).

Accès : La première grande catégorie de produits offerte par EURODICAUTOM est l'interrogation par lots ou en mode conversationnel. L'interrogation par lots ${ }^{3}$ est faite par des terminologues à partir de textes à traduire que le traducteur a marqués, c'est-à-dire que les termes représentant une plus grande difficulté d'ordre traductionnel ou terminologique y sont signalés. En retour, le traducteur reçoit une liste d'équivalents aux termes qu'il avait désignés. Il est aussi possible d'émettre des listes de termes ou d'unités phraséologiques par domaine, par langue, par bureau émetteur etc., dans le cadre de projets de traduction particuliers. En mode dialogual ${ }^{4}$, qui est le type d'utilisation favorisé par la direction de la banque, il y a de même possibilité de limiter la recherche par domaine, par langue, par cote de qualité, etc.

Autres produits : D'emblee, EURODICAUTOM servit à la publication de glossaires, prenant ainsi la suite du DICAUTOM qui, selon son principal artisan (Bachrach 1981:28), eut comme seule utilité la production de quatre glossaires phraséologiques 
multilingues sur les thèmes des quatre congrès de l'acier tenus à Luxembourg de 1964 à 1968. On a intégré un programme de photocomposition à Eurodicautom 2 afin de faciliter la production rapide de lexiques ou de glossaires par domaine, ou par projet.

Mais il existe aussi toute une famille d'autres produits dont on fait plus rarement mention parce qu'ils concernent la gestion de la banque. Ainsi, il va presque de soi que la banque possède les facilités nécessaires à la mise à jour et à la modification des données présentes dans ses mémoires. Un logiciel destiné à la production de statistiques y a aussi été intégré. On y procède de plus à la production de bandes et disques magnétiques destinés aux échanges d'informations inter-banques et, en contrepartie, certains sous-systèmes peuvent y traiter les bandes et disques magnétiques importés. On y a enfin mis au point un module de recherche de la redondance qui produit des rapports grâce auxquels on remédie au problème (François 1981 : 157).

\section{Réseau de diffusion}

L'accès à EURODICAUTOM est gratuit pour tous ceux qui obtiennent le privilège de joindre son réseau de diffusion. Le service vise d'abord les quelque 2000 langagiers (linguistes, rraducteurs, interprètes et terminologues) des institutions communautaires et/ou européennes. Le réseau comprend ensuite divers abonnés, bibliothécaires, langagiers pigistes, linguistes et professeurs de langue, institutions d'enseignement, etc., qui se branchent à la banque grâce à ECHO (European Commission Host Organization), serveur officiel de la Commission et représentant de la banque dans le réseau télématique EURONET-DIANE (EURONET étant le réseau de télécommunications parrainé par la $\mathrm{CCE}$, tandis que DIANE - Direct Information Access Network for Europe - regroupe des banques et serveurs indépendants) (Iljon 1981: 151). EURODICAUTOM devint accessible par ECHO dès la création du service en 1980. Depuis, son réseau de diffusion n'a pas cessé de croître. Quand ECHO débute ses activités en juin 1980, douze clients se montrent intéressés par EURODICAUTOM; en août 1981, ECHO compte cent quatre clients dont quatre-vingt-quinze s'intéressent à la banque de terminologie. Et s'il n'en coûte rien d'interroger la banque, le serveur, de son côté, facture l'exploitation de ses systèmes: l'utilisateur doit donc payer au moins son temps de connexion (Iljon 1981 : 151-152). Selon des données recueillies en 1987, la banque traitait une moyenne quotidienne de 2000 questions posées par les utilisateurs des institutions communautaires ou européennes, et de trois cents par les abonnés extérieurs (Quicheron 1988 : 149).

\section{Conclusion}

On s'entend généralement pour dire que la banque de la CCE a été mise au point par des traducteurs pou des traducteurs. Le DICAUTOM puis EURODICAUTOM furent conçus en vue d'abolir les difficultés de la recherche terminologique des tâches du traducteur dans le contexte particulier des institutions communautaires et européennes. Mais l'aspect traductionnel de même que les visées proprement terminologiques furent peu à peu subordonnés à un impératif de justesse juridique: les textes qui proviennent de la $\mathrm{CCE}$, en traduction ou dans leur langue source, font office de standard, voire de norme jurilinguistique. En conséquence de quoi on a toujours accordé une importance vitale à la mention des sources dans EURODICAUTOM. Et le problème s'embrouille d'autant que l'on doit maintenant tenir compte de neuf langues dont, en principe, le statut est égal. On perçoit quelles difficultés cela représente en matière de terminologie: questions d'équivalence, écart référentiel par rapport au pivot notionnel d'une langue à l'autre, etc. Enfin, dernière remarque digne de mention, Hotz (1983:50) met au jour une parenté étroite entre la conception et le fonctionnement d'EURODICAUTOM et de TEAM, la banque de Siemens.

\section{LES BANQUES ALLEMANDES}

Après la présentation de leur contexte d'origine et les explications de leurs fondateurs respectifs, LEXIS et TEAM seront décrites par le biais de leur contenu et de leur mode d'exploitation. On tracera ensuite le plus exactement possible un profil de leur réseau de diffusion, malgré la rareté des informations disponibles à ce sujet. Dans le cas de TEAM, enfin, il sera aussi question des développements récents, c'està-dire de ceux survenus depuis quelques années.

\section{LEXIS}

Situation

Des recherches effectuées en République fédérale d'Allemagne depuis le début des années 1960 conduisirent les dirigeants du Service de traduction rattaché au ministère de la Défense à remettre en question certaines de leurs méthodes de travail (Krollmann 1971 : 117-118). Portant sur la traduction technique, ces recherches de la Federal Armed Forces Translation Agency devaient révéler le moyen d'atteindre quatre objectifs :

1) améliorer la qualité de la traduction (réduire la marge d'erreur);

2) augmenter la productivité des traducteurs (diminuer les coûts);

3) accélérer la publication des traductions ;

4) uniformiser la terminologie.

La précision, la rapidité, la fiabilité et l'uniformité du travail de traduction furent donc plus particulièrement étudiées.

Les résultats qu'on obtint furent en partie publiés dans le numéro 5 (janvier 1965) de Beitrage zur Sprachkunde und Informationsverarbeitung. Il en ressortait 1) une nette corrélation entre, d'une part, le nombre et le genre d'erreurs commises et, d'autre part, le degré de difficulté des textes; 2) un rapport direct entre le temps de traduction et la technicité des 
textes, attribuable aux difficultés terminologiques (Hotz 1983: 9). C'est ainsi qu'on en vint, dans l'armée fédérale allemande, à concevoir un projet de banque de mots afin d'assister les traducteurs spécialisés dans l'accomplissement de cette partie ardue de leur tâche (Rondeau 1981 : 164). On souhaitait améliorer le rendement des traducteurs techniques de $50 \%$ (Goetschalckx 1968: 50). Cette banque de mots allait porter le nom de LEXIS (pour Lexikographisches InformationsSystem, en français: service d'information lexicographique). Elle est mise sur pied à partir de 1966, à Mannheim, où on emmagasine une première tranche de 120000 termes. Quelques années s'écoulent avant que le système soit opérationnel et que LEXIS passe, en 1969, sous l'autorité d'un nouvel organisme, le Bundessprachenamt (ou Office fédéral des langues). Le changement administratif s'accompagne d'ailleurs d'un changement géographique et LEXIS déménage à Hürth.

Au départ, LEXIS devait permettre l'amélioration du rendement des traducteurs en mettant à leur disposition et en centralisant toute l'information terminologique dont ils étaient susceptibles d'avoir besoin. L'Office fédéral des langues (OFL) ajoutera à cette préoccupation première avec les années: l'enseignement des langues de spécialité, la normalisation terminologique, la coopération internationale en matière de terminologie.

\section{Ressources terminologiques}

Les informations terminologiques consignées dans LEXIS proviennent des traductions de nature technique (manuels d'instructions d'appareils militaires, documents juridiques, dossiers relatifs à des projets internationaux) effectuées à l'OFL (Hotz 1983: 7). De longueur variable, les termes relevés et enregistrés appartiennent à des domaines spécialisés, le plus souvent scientifiques ou techniques; il s'agit à l'occasion de néologismes pas encore répertoriés dans les supports d'information traditionnels. Ils se présentent dans une forme la plus dépouillée possible, c'est-à-dire qu'ils ne comportent pas «de signes diacritiques, de notes grammaticales, de rapports hiérarchiques, de notes relatives à l'homonymie ou à la synonymie ainsi que, le plus souvent, de contextes ou de définitions» (Rondeau $1981: 164$ ).

Ce qu'on identifie par entrée dans LEXIS est en réalité une paire de termes accompagnée d'une série de codes. À part l'allemand, langue pivot de la base de données, une entrée peut comporter de l'anglais, du russe, du français, du néerlandais, du portugais ou de l'italien. Idéalement, la moitié allemande de l'entrée serait normalisée, mais dans les faits, cela ne représente qu'une faible proportion de la banque. Les informations terminologiques sont classées en deux fichiers inversés : dans le premier, la langue d'accès à l'information est l'une des six autres langues appariées à l'allemand dans le second, c'est par l'allemand qu'on accède aux paires de termes. Chacun des deux fichiers comportait 1434000 entrées (paire de termes) au début des années 1980 (Krommer-Benz 1985 : 62).
La terminologie est classée par domaines selon un thésaurus bilingue, le TEST (Thesaurus for Engineering and Scientific Terms) conçu par le ministère américain de la Défense, mais traduit et révisé par l'Institut allemand de la documentation (Krollmann $1971: 122)$. Ce thésaurus comporte environ 250 domaines génériques.

En plus de l'information minimale propre aux entrees de LEXIS, et à l'encontre de ce qu'affirme Rondeau ci-dessus, des informations supplémentaires (définitions ou contextes, termes génériques ou spécifiques, antonymes ou synonymes) peuvent documenter les notions; elles sont alors enregistrées dans une mémoire auxiliaire, LEXIS II (De Bessé 1983: 168), où elles sont accessibles dans un deuxième temps (Hotz 1983: 22).

\section{Exploitation}

LEXIS a été conçue et est utilisée comme aide à la traduction. C'est ce qui explique son contenu, sa structure et qui devrait en principe justifier la manière dont on l'exploite.

Personnel : En 1980, Sager et McNaught (p. 24) rapportaient que dix-huit terminologues étaient affectés à temps plein à l'entretien et à l'amélioration des fichiers de LEXIS. Dix d'entre eux étaient chargés de l'alimentation et de l'approvisionnement en nouveaux termes, tandis que les autres répondaient aux demandes ponctuelles, siégeaient à des comités ou poursuivaient d'autres activités. Leur production moyenne variait de vingt-cinq à trente paires de termes par jour et leur contribution totale à l'enrichissement des fichiers représentait environ 40000 paires de termes par année. Les traducteurs de 1'OFL n'étaient pas mis directement à contribution, mais c'est grâce à leurs demandes qu'on repérait de nouveaux termes et qu'on identifiait de nouveaux champs de recherche terminologique. Leurs commentaires et les corrections qu'ils signalaient à l'occasion faisaient partie du flux d'informations sur lequel comptait la direction de la banque.

Produits : À partir des programmes de LEXIS on peut produire des listes alphabétiques par domaines, par projets, par sources d'information, etc. On peut aussi publier des dictionnaires ou des glossaires, alphabétiques ou systématiques. À cet égard, d'ailleurs, l'OFL recevait environ 500 demandes annuelles de dictionnaires spécialisés au début des annés 1980 Mais le produit principal de la banque demeure le text-oriented glossary, liste bilingue qui suit l'apparition des termes difficiles dans un texte de départ et qui fournit au demandeur un équivalent dans la langue cible. Les traducteurs de l'OFL reçoivent aussi des microfiches, mises à jour annuellement, qui reproduisent tout le contenu des fichiers de la banque. Un programme permet enfin d'obtenir diverses informations statistiques utiles surtout aux linguistes et aux professeurs de langue (Krollmann $1971: 123$ ).

Modes d'accès : LEXIS est accessible en différé, dans le sens où ceux qui profitent de son contenu, les traducteurs de l'OFL, doivent adresser leurs demandes 
aux terminologues de la banque. Ces derniers sont les seuls à y avoir un accès direct. C'est d'ailleurs ainsi qu'ils enregistrent de nouvelles données, qu'ils corrigent les entrées où on a relevé des erreurs, qu'ils éliminent de l'information devenue inutile ou superflue. Pour la consultation, le repérage peut se faire par terme, par domaine, par segment morphologique, c'est-à-dire que la recherche peut porter sur une chaîne de caractères au moyen de troncations antérieures ou postérieures. La consultation de l'index est aussi possible. Il convient enfin de mentionner que LEXIS a été dotée dès le départ d'un programme destiné à éliminer la redondance : le lendemain même de leur stockage en banque, un relevé des termes redondants est émis à l'intention des terminologues.

De plus, LEXIS comporte un système de photocomposition, Digiset, qui permet de produire des publications, dictionnaires ou lexiques, avec une présentation comparable à celle d'ouvrages publiés par des éditeurs professionnels. Ces publications font suite aux demandes adressées à l'OFL par le ministère de la Défense, par d'autres ministères ou organismes gouvernementaux ou même, moyennant paiement, par des entreprises ou organismes externes.

On étudie enfin depuis quelques années la possiblité de rendre la banque disponible sur DOC (Célestin 1990).

\section{Réseau}

Les terminologues et traducteurs de l'OFL forment le cœur du réseau de diffusion terminologique de LEXIS. En 1981, ce groupe comptait 150 traducteurs plus précisément affectés aux travaux du ministère de la Défense (Mouzard: 5). Les autres ministères et organismes gouvernementaux de l'exRFA y participaient aussi, mais dans une moindre mesure.

LEXIS rejoint une clientèle souvent ignorée des banques de terminologie, soit celle des professeurs de langue: ceux de l'OFL, spécialisés dans les langues de spécialité, y ont recours avec assiduité. Il faut aussi signaler que les terminologues de l'OFL sont très présents aux divers comités de normalisation, assurant par là un moyen supplémentaire de diffuser l'information terminologique consignée dans la banque. Signalons enfin que quelques entreprises et des centres d'information ou de documentation indépendants y ont également accès (en différé, selon toute vraisemblance) en payant les services rendus.

\section{TEAM}

\section{Situation}

Il convient de préciser d'entrée de jeu que Siemens AG, la société qui a conçu et qui administre TEAM, est une multinationale faisant des affaires dans de nombreux pays d'Europe, d'Afrique, d'Amérique et d'Asie. Ses activités sont concentrées dans les industries de pointe comme l'électronique, les télécommunications, l'informatique, etc. L'entreprise produit un million de pages de documentation par année (Hvalkof 1982:7), dans la langue parlée ou comprise par son client. D'autre part, Siemens vend aussi bien des ordinateurs géants que des appareils téléphoniques; l'acheteur peut avoir besoin d'un manuel technique détaillé et précis de plusieurs centaines de pages qui dépasse largement le seuil d'intelligibilité du lecteur moyen, tout comme il peut se satisfaire d'une brochure explicative de quelques paragraphes où sont expliqués en termes simples les traits distinctifs d'un appareil courant. La société s'adresse donc à une clientèle disparate qui possède des compétences techniques, scientifiques et linguistiques très diversifices.

A l'image d'autres empires industriels, la société Siemens investit dans des projets qui présentent de raisonnables perspectives de rentabilité. $\grave{A}$ la suite de la décision prise au ministère de la Défense de se doter d'une banque de mots, des coûts sans cesse croissants de la production linguistique (rédaction technique et publicitaire, traduction) dans l'entreprise (Hotz 1983: 26), à la suite également de sa propre évaluation de la rentabilité d'un tel système (Schulz 1971: 123), la direction des services linguistiques de Siemens mit en marche le projet d'une banque de terminologie en 1967, à Munich. On la nommerait TEAM, pour Terminologie-Erfassungs- und Aus wertungs- Methode (méthode de saisie et d'exploitation de terminologie) et lui fixerait quatre objectifs :

1) aide aux traducteurs de la société et de ses associés ;

2) aide aux rédacteurs techniques de la société ;

3) stockage intégral et gestion des données terminologiques utiles à l'ensemble de la société ;

4) regroupement de la documentation destinée à des cours de langue de spécialité (Hohnhold $1984: 20$ ).

Tout laisse croire que la direction de Siemens poursuivait un autre objectif: pour une entreprise qui convoite la plus grande part possible du marché mondial de l'informatique et des télécommunications, une banque de terminologie peut représenter un banc d'essai exceptionnel, un objet de recherche et d'expérimentation unique. On peut y étudier comment, par exemple, l'informatique et les télécommunications peuvent se combiner dans le traitement plus ou moins automatisé des langues, on peut y expérimenter et y mettre au point des systèmes rentables que l'on commercialisera ensuite, on peut s'y appliquer à résoudre des problèmes dont les solutions seront généreusement subventionnées par des organismes internationaux (comme la collaboration avec EURODICAUTOM, le traitement de l'arabe à partir du début des années 1980 , etc.).

\section{Ressources terminologiques}

Vers 1980-1981, TEAM contenait plus d'un million de dossiers (ou fiches). Mais ce total ne représentait pas que des fiches terminologiques, puisque TEAM compte aussi des dossiers bibliographiques et encyclopédiques (Rondeau 1981 : 162). Il est toutefois 
permis de croire que ce dernier type d'information occupait une faible proportion des fichiers de la banque. En tout état de cause, le million de dossiers correspondait à une somme de termes évaluée à trois ou quatre millions. Toutes les fiches terminologiques comportent de l'allemand ainsi qu'une autre langue au moins. L'anglais est traité sur $70 \%$ des fiches, le français sur $40 \%$, l'espagnol sur $30 \%$, les autres langues sur $20 \%$. La fiche terminologique de TEAM, appelée entry, a une longueur variable. Elle peut comporter des informations lexicales des plus élémentaires comme elle peut contenir des passages complets de notices techniques, le tout réparti sur 100 champs (data categories). En vedette, on retrouve des unitermes, des pluritermes ou des unités phraséologiques (Hohnhold 1984: 23 et ss.). Les fiches sont le plus souvent constituées en pools, regroupements autour d'un code de rédacteur, d'un code de produit ou en fonction d'un même bureau émetteur, division de Siemens ou société extérieure, etc. (Brinkman 1981 : 69; Hohnhold 1984 : 24 ; Schulz $1980: 222$ ).

Pour le classement des données, Siemens a adopté le code de classement mis au point par la Dokumentationsring der Elektroindustrie, l'Union de documentation de l'industrie électrique allemande (Schulz 1971 : 126). Après une adaptation initiale, on a continué à le façonner aux besoins particuliers de TEAM (Krommer-Benz 1985: 66). Les principaux domaines traités dans la banque ont rapport à I'économie (finance, comptabilité, etc.) et à la technologie (électronique, informatique, télécommunications, etc.).

Malgré l'étroite collaboration qui unit TEAM au Deutsches Institut für Normung, la normalisation terminologique ne représente aucune contrainte selon les porte-parole de la banque; ils recourent plus volontiers aux notions de standardisation ou d'uniformisation.

\section{Exploitation}

Personnel : En 1980 (Sager et McNaught : 24), le personnel directement affecté à l'entretien de la banque se composait de sept analystes, neuf terminologues, trois lexicographes, quatorze préposés au traitement et à la saisie des données, quatre assistantsterminologues/lexicographes à temps partiel. Ce groupe faisait interface entre TEAM, d'une part, et les quelque 250 traducteurs permanents et la centaine de pigistes de la société, d'autre part. La direction de Siemens estimait à ce moment-là qu'un terminologue pouvait produire un minimun de 3333 nouveaux termes par an et, compte tenu de toutes les activités auxquelles se consacraient leurs terminologues, que les fichiers de TEAM devaient s'enrichir d'au moins 15000 nouvelles fiches par an.

Produits: TEAM est évidemment dotée de programmes permettant d'émettre des listes alphabétiques unilingues, bilingues ou multilingues, d'entrées lexicales ou phraséologiques, par domaine ou par projet. Sur demande, on obtient également des index unilingues par domaines ou par projets, des glossaires contextuels, des text-oriented glossaries comme décrits dans LEXIS mais que l'on appelle ici des textrelated ou text-synchronous lists (Hohnhold 1984: 28). Le système peut aussi compiler des données statistiques. Un système de photocomposition intégré permet de plus à Siemens de produire diverses publications terminologiques en différents formats; en employant les alphabets latin, cyrillique ou les caractères de l'arabe, sur support papier ou film offset. Des microfiches ou des microfilms peuvent enfin être émis à partir de n'importe quelle partie des fichiers. Il s'écoule un maximum de six semaines entre la décision de produire une publication et son aboutissement (Brinkman 1981 : 73).

Modes d'accès: On peut accéder à TEAM en différé, soit pour la production d'ouvrages lexicographiques ou terminologiques, soit à partir d'une liste de termes: la demande est adressée à un terminologue qui voit à satisfaire la demande du traducteur, de l'éditeur ou du rédacteur.

On y accède aussi en direct. Au moment de l'interrogation, la recherche peut porter sur des termes, leurs synonymes, abréviations, etc., sur une chaîne de caractères (possibilité de troncation postérieure), sur des domaines, des codes de projet, des codes de source, etc. (Schulz 1980: 223-224). L'index (autant celui de toute la banque que celui de chacun des pools) peut de même être consulté dans la langue source (Hohnhold $1984: 29$ ). On peut en fait accéder à la terminologie en partant de n'importe quelle langue. En ce qui concerne les publications qui nécessitent des travaux de photocomposition, l'équipe de TEAM peut les préparer, à l'intention du demandeur, directement à l'écran. De la même manière, la mise à jour, l'alimentation, les corrections se font en direct. TEAM est enfin dotée d'un programme qui permet de combiner la traduction et l'accès direct à la base de données : dans ce contexte, le traducteur travaille face à un écran où apparaissent en surimposition la langue source et la langue cible tandis que l'accès à la base de données se déroule dans une fenêtre.

Le contenu des fichiers de TEAM peut enfin être fouillé automatiquement: un texte de départ déjà enregistré sur ruban magnétique est acheminé vers un programme de repérage automatique qui, en regard de chaque terme du texte, propose un (ou des) équivalent(s) dans une langue cible prédéterminée.

Réseau

Le réseau de diffusion de TEAM est d'abord constitué par les langagiers de la société Siemens. Cela a d'ailleurs toujours été un objectif important de la société : que tous ses employés qui doivent manipuler des concepts en vue de la recherche ou du développement, de la mise en marché ou de la publicité, puisent à une source unique d'information. C'est pourquoi toutes les succursales de la société, partout au monde, sont en principe intégrées au réseau. Siemens a de plus greffé les entreprises qui lui sont associées à ce réseau de diffusion terminologique.

Quant au réseau externe, il se compose d'un certain nombre d'organismes nationaux ou interna- 
tionaux, d'universités et d'entreprises qui paient leur accès aux ressources de la banque. Les cabinets de traduction et les traducteurs pigistes qui font affaire avec Siemens peuvent profiter de TEAM soit en direct, soit en différé : on leur remet des listes de termes jumelées aux textes à traduire, les text-synchronous lists (Brinkman 1981; 72). Pendant des années, on pouvait appartenir au réseau de diffusion externe de TEAM en offrant des données terminologiques ou lexicographiques en échange de l'accès aux informations terminologiques de Siemens. D'ailleurs, les porte-parole de la banque parlaient volontiers d'entreprise cooperative: on offrait aux sociétés et organismes du réseau externe de constituer leur propre pool qu'ils traitaient eux-mêmes ou qu'ils confiaient aux techniciens de Siemens (Brinkman 1981: 71; Schulz $1980: 222$ ). Mais au cours des dernières années, cette formule, apparentée au troc, a été remise en question puis peu à peu remplacée par une relation client-fournisseur où le client paie simplement au fournisseur une prestation de service.

\section{Évolution récente}

Il importe de signaler comme particularité de TEAM son évolution constante et harmonieuse, sans heurts, qui a accompagné assidûment le développement de l'informatique. Tant dans son matériel que dans ses logiciels, tant dans sa méthode de classemen que dans ses modes d'accès, TEAM a suivi de près l'évolution de la technologie informatique en général et celle des SGBD en particulier.

On a par ailleurs entrepris la mise en marche d'un logiciel de traduction automatique interactif, METAL, qui emploie la terminologie de TEAM et qui, en situation normale d'utilisation dans la société, est raccordé directement à la base de données, dans le sens où la dimension lexicale et terminologique du texte d'arrivée est comparée automatiquement au contenu de la banque (Bennett et al. 1986: 45 ; Brinkman 1981 : 74). Enfin, Siemens a résolu de surmonter les divers problèmes inhérents à la constitution d'un réseau externe de diffusion terminologique en commercialisant son produit sous forme de mini-banque, le système Term-PC, progiciel de gestion de base de données terminologiques unilingue ou multilingue qui accepte facilement de nouvelles données, les mises à jour, mais aussi les ensembles de fiches terminologiques vendus en blocs de dix disquettes par Siemens. La société soutient qu'il s'agit d'un moyen de faciliter les échanges entre TEAM et les langagiers (Siemens 1989 ; Vollnhals $1989: 20-23$ ).

\section{Conclusion}

L'intérêt des deux banques décrites tient, au-delà de leur situation géographique et de la singularité linguistique que cela leur conferre (les deux accordent une place prépondérante à la langue allemande), dans le fait qu'elles ont toutes les deux survécu un quart de siècle. Ce qui implique de nombreuses difficultés surmontées et tout autant de problèmes résolus. Il est permis de croire que leurs objectifs initiaux, simples et cohérents, ont probablement contribué à une évolution aussi rectiligne. LEXIS et TEAM furent mises sur pied par des organismes qui traitaient un imposant volume de textes à traduire et qui, en conséquence, embauchaient de très nombreux traducteurs (plus d'une centaine dans chacun des cas). Tant a l'OFL que chez Siemens, la technicité des textes faisait obstacle à un rendement optimal des traducteurs et provoquait un flottement inacceptable dans la terminologie employée. Les deux banques devaient donc améliorer le rendement des traducteurs et uniformiser la terminologie.

LEXIS et TEAM ont en commun qu'elles sont employées dans des cours de langues de spécialité. En vingt-cinq ans (et plus...), elles ont toutes les deux accumulé un contenu terminologique très volumineux. Signalons enfin qu'elles favorisent l'accès en différé plutôt que l'accès direct, bien que les raisons invoquées diffèrent dans l'un et l'autre cas. Elles privilégient la diffusion terminologique en réseau interne plutôt que d'exploiter prioritairement un réseau externe.

Elles se distinguent cependant sur deux points. La structuration de leurs informations terminologiques ne se ressemble en rien. Enfin, tandis que LEXIS a mis sur pied un système inchangé en vingt-sept ans, TEAM s'est toujours conformée à l'évolution de la technologie informatique.

\section{LES BANQUES CANADIENNES}

Malgré ce titre, l'origine des deux banques dont il sera maintenant question est entièrement attribuable au poids démographique, politique et économique du Québec, aux contraintes que cette province a imposées au reste du pays en matière linguistique. De plus, tant la BTQ que TERMIUM furent mises sur pied au Québec par des chercheurs québécois. En fait, ce sont des choix politiques prenant appui sur des circonstances historiques et socio-économiques qui allaient créer une situation propice à la mise sur pied de banques de terminologie destinées à répondre aux exigences du marché québécois.

Tandis qu'en Europe la conception des premiers prototypes de banque de terminologie accompagnait l'élaboration d'une science terminologique par des theoriciens comme Wüster, deux facteurs allaient favoriser leur apparition au Québec. Comme partout ailleurs, la multiplication des échanges commerciaux jumelée aux progrès techno-scientifiques vont entraîner la formation locale d'un milieu de la traduction. Ce dernier prendra forme lentement: peu à peu, les traducteurs vont se regrouper, s'organiser, se concerter. C'est dans ce milieu, animé par le Journal des traducteurs et dont le noyau est situé à l'Université de Montréal, qu'on prend d'abord conscience de la nécessité d'une démarche, de méthodes et d'activités terminologiques.

La résurgence du nationalisme québécois dans les années 1960 peut certainement être évoquée comme second facteur en ce qu'il a contribué de manière prépondérante aux revendications politiques des francophones. Ayant épousé la cause de la langue 
française, ce nationalisme allait engendrer des remous dans les secteurs politiques et culturels, il allait s'attaquer aux infrastructures économiques mises en place et exploitées très majoritairement par des anglophones.

Les technologies se sont propagées en anglais et c'est cette langue qui a longtemps dominé dans le monde du travail, du commerce et des affaires. Même lorsque la langue du travail était le français, il était courant de constater un usage massif de terminologies anglaises dans de très nombreux secteurs d'activités.

Ce rapport de forces entre le français et l'anglais est devenu de plus en plus gênant avec la montee des francophones qui, depuis la fin des années cinquante, ont assuré progressivement la prise en charge de tous les secteurs de la société et plus particulièrement dans l'économie. (Rousseau $1990: 68$ )

De rencontre en organisation, de réunion en association, les langagiers (avant la lettre) se retrouvent au colloque de Stanley House sur la traduction, organisé par le Conseil des arts du Canada en 1965. La STIC (Société des traducteurs et interprètes du Canada), la STIO (Société des traducteurs et interprètes de l'Ontario), la STM (Société des traducteurs de Montréal), le Bureau fédéral des traductions, la Société Radio-Canada, les départements de linguistique de l'Université de Montréal et de l'Université Laval, l'Office de la langue française et le Service régional de la langue française à Amos (Darbelnet 1965: 329) y sont tous représentés. Une proposition généralement considérée comme la première mention publique d'un projet de banque de terminologie au Canada $y$ fut soumise. Pour Jean-Paul Vinay, celui qui lance l'idée, une centrale terminologique viserait à satisfaire le besoin de rassembler et diffuser les informations terminologiques requises, enregistrées ou produites par les représentants des organismes présents ou par d'autres.

L'Office de la langue française venait tout juste de créer un centre de terminologie et avait reçu le mandat de définir la norme du français parlé et écrit au Québec (Darbelnet 1965: 330). D'autre part, le Bureau des traductions du Secrétariat d'État, avec des équipes réparties aux quatre coins du pays (à Ottawa, Montréal, Québec et Toronto surtout), se ressentait d'une pareille dispersion des efforts et des crédits, d'une telle duplication de la recherche (Gobeil 1981: 8). Aussi la proposition de Jean-Paul Vinay consistant à mettre sur pied une centrale de terminologie informatisée tombait-elle à point nommé (Clas 1969: 191); il suggérait en outre que l'Office de la langue française coordonne tous les efforts de centralisation, ou à tout le moins que tout projet de ce type soit élaboré en étroite liaison avec l'Office (Darbelnet 1965: 330).

Le projet de Jean-Paul Vinay fit cependant long feu. Trois ans plus tard, lors d'un autre colloque sur la traduction organisé par l'Office de la langue française, André Clas, de l'Université de Montréal, relance l'idée d'un fichier automatique de terminologie autofinancé, qui se consacrerait à la solution de problèmes de traduction (Dubuc et Grégoire $1974: 181$ ).

À la suite de cette communication, le conférencier fut chargé par les participants du Colloque d'entreprendre les démarches nécessaires pour concrétiser la proposition présentee. L'intéressé convoqua en janvier 1969 une «assemblée» et «quarante sages» répondirent à l'appel. Cette assemblée décida de former un comité d'étude et, en mars de la même année, le rapport soumis par ce comité fut adopté (Meta 14-4: 191-194). Parallèlement, le rapport du comité fut entériné par le Conseil de la faculté des lettres et remis aux autorités de l'Université pour en saisir les gouvernements provincial et fédéral. Une entente tripartite, Secrétariat d'État du Canada, ministère des Affaires culturelles (OLF) et Université de Montréal, fut conclue. (Clas $1987: 212$ )

En parallèle, les activités, rencontres et discussions vont se multiplier. En mars 1969, l'Office de la langue française réorganise son centre de terminologie, le consacrant désormais à l'étude des vocabulaires. Puis, avec le concours du Centre de documentation de l'Université Laval, l'organisme conçoit un programme de traitement électronique de l'information terminologique et entreprend la constitution d'un fichier terminologique semi-automatisé (Kerpan 1977 : 48-51 ; Rondeau 1981 : 156). À l'hiver 1970, un colloque sur la situation du français au Québec est organisé par le Bureau régional de la diffusion du français de la ville de Québec: on y propose la création immédiate d'une banque de terminologie en vue de diffuser la norme du français au Québec telle que définie par l'OLF (Dubuc 1970 137-139). C'est aussi à ce moment que 1'OLF, soucieux de coordination, commence à dresser un inventaire annuel des travaux de terminologie, d'abord au Québec, puis en France. Pour passer à un nouvel état de langue, on songe à un système où le français serait favorisé et dont la vocation serait informative, normative et éducative (Hotz 1983: 57-58). À l'automne 1971, l'OLF amorce une collaboration avec EURODICAUTOM et LEXIS.

Ailleurs, hors des cercles de la traduction et de la terminologie, divers groupes exercent des pressions politiques telles que les instances gouvernementales tant fédérale que provinciale se voient contraintes d'adopter des législations linguistiques. Il fallait en effet trouver les moyens de faire plus de place au français dans le monde du travail, du commerce et des affaires. Et pour assurer la francisation, on avait besoin de mettre à la disposition des entreprises et de l'administration une terminologie française fonctionnelle qui garantisse la poursuite quotidienne des activités (Fortin 1978a : 1; Fortin 1988: 51-52) : il fallai démontrer que cette terminologie existait et qu'elle 
pouvait se révéler aussi efficace que l'anglais à l'usage. Pour le reste, on formulait le projet de rendre les ressources terminologiques de la francophonie utilisables par les Québécois et de créer par la même occasion une centrale de terminologie qui deviendrait un lieu d'échange terminologique local (Fortin 1978a: 1-2; Fortin 1984: 201-202).

\section{TERMIUM}

Situation

À la suite des travaux du comité d'étude présidé par André Clas, la BTUM (pour Banque de Terminologie de l'Université de Montréal) est officiellement inaugurée le 2 octobre 1970 avec l'idée d'en faire le fichier bilingue le plus souple qui soit (Dubuc et Grégoire 1974 : 181 ; Adshead 1985 : 64). L'instrument devra servir à la recherche et à la documentation en terminologie et en traduction aussi bien qu'à des fins pédagogiques (Clas 1971: 5). On lui assigne les objectifs suivants :

1) désignation des produits de la technologie ;

2) mise en commun des ressources et organisation systématique de la recherche terminologique;

3) centralisation de l'information terminologique pour qu'il soit ainsi plus facile de la traiter et de l'épurer ;

4) orientation de la recherche dans des directions données;

5) disponibilité pour tous les intervenants du milieu (Clas 1969: 192).

On insiste d'ailleurs sur ce demier point: pour que le dictionnaire automatique profite à tous, pour qu'il bénéficie d'un appui unanime et qu'il ne soit pas manipulé par un groupe en particulier, les forces vives de la société en matière linguistique doivent toutes s'y impliquer (Clas 1969: 192-193),

Dans sa conception, la BTUM doit permettre un accès direct par terminal aux utilisateurs qui ont à la consulter rapidement en cas de besoin; elle doit pouvoir compiler des index alphabétiques quand on y regroupe des informations terminologiques disparates; les corrections et les mises à jour doivent pouvoir s'y faire directement à partir du terminal afin qu'on ait accès à une information de pointe (Dubuc et Grégoire 1974: 182). Or l'informatique offre toutes ces possibilités : stockage massif de l'information, accès direct et facilité d'ajouter ou de corriger des données (Dubuc et Grégoire 1974: 181). La banque serait initialement financée par des subventions gouvernementales et privées; elle tirerait ensuite ses revenus d'abonnements et de profits sur les publications extraites de ses fichiers. Les services qu'elle offrirait prendraient diverses formes: exemplaire annuel du trésor terminologique, glossaires spécialisés, consultations téléphoniques, bulletin mensuel et service de publication (Clas 1969: 192).

Le premier directeur de la BTUM est Marcel Paré. À son avis, une pareille entreprise doit remplir un mandat beaucoup plus vaste que ses stricts objectifs terminologiques :

Par l'entremise des traducteurs, journalistes, publicitaires et éducateurs qui la consulteront, la Banque espère contribuer entre autres à enrichir la langue du travail au Québec. (Coty $1970: 195$ )

Dans les années qui suivent, l'élaboration de la banque se fait en collaboration avec le milieu de la traduction. Fin 1974, on s'estime prêt à passer du stade expérimental à celui de l'exploitation (Marchand 1976a: 173). Aussi, en 1975, on procède à une première expérience de liaison BTUM-entreprise (les services linguistiques de Bell Canada) afin d'obtenir diverses statistiques et de connaître les réactions des utilisateurs. En septembre de la même année, on expérimente la première consultation intercontinentale d'une banque de terminologie : la BTUM est alors interrogée à partir de l'Europe (Couture 1976: 100 et ss.).

Pendant ce temps, depuis la fin de 1974, le Bureau des traductions du Secrétariat d'État du Canada a confié à un goupe nommé Mission Terminologie le soin d'étudier les possibilités d'exploiter au mieux les ressources linguistiques et terminologiques que le Bureau pouvait rassembler (Larose 1976:1) et de découvrir les moyens de normaliser la terminologie anglaise et française employée dans l'administration publique fédérale et dans les corps publics (Gobeil 1981:8). Le Bureau avait déjà réuni 125000 fiches bilingues sur le potentiel de 1400000 qu'on calculait avoir consignées en trente-cinq ans de travaux de traduction, le reste demeurant éparpillé dans les quatre-vingt-dix sections et sous-sections du Bureau (Cardin 1984a: 18; Kerpan 1977: 51). Mission Terminologie propose de recourir à une banque de terminologie comme principal moyen d'atteindre les objectifs de normalisation et d'exploitation. Aussi, dès janvier 1976, le Secrétariat d'État acquiert la BTUM. Le système est alors transféré sur l'ordinateur central du ministère de l'Énergie, Mines et Ressources à Ottawa (Adshead 1985: 64; Gobeil $1981: 8$ ).

On modifie le logiciel TERMIUM (pour TERMInologie Université de Montréal), qui devient alors TERMIUM 1 , on achève de rassembler les fiches de traduction du Bureau, et le Secrétariat d'État peut alors se consacrer à sa première priorité, soit ajouter ces fiches aux quelque 175000 que contenait déjà la BTUM (Marchand 1976a: 174-175). C'est ainsi qu'on cumulera d'abord plus de 1200000 fiches dans TERMIUM 1 (Cardin 1984a: 18). Après les opérations de tri, de classement, de mise en forme et de saisie, on allait devoir passer avec TERMIUM 2 à la phase d'épuration, fin 1978 (Andersen 1982 : 8). On obtient alors 800000 fiches, puis 750000 , et ce nombre ira en diminuant quelque temps encore avant de recommencer à augmenter: 600000 en 1980 1981,784000 en 1985, puis 900000 en septembre 1987 (Larose 1976: 2-3; Gobeil 1981: 9; Cardin 1984a : 18; Rondeau 1984: 168; Adshead 1985: 64 ; Wells $1988: 58$ ). 
Mais dès 1981 , on entreprend déjà de définir et concevoir ce que serait TERMIUM 3 . On projette alors de simplifier l'organisation des données en constituant des fichiers complémentaires intégrés (fonds multilingue, fichier documentaire, fonds des difficultés de traduction, etc.). L'étude d'un nouveau système est alors justifiée par l'expansion rapide du réseau et par les limites techniques de TERMIUM 2: on souhaite se doter de matériel plus perfectionné, implanter un nouveau logiciel et passer à un système de troisième génération. TERMIUM 3 est défini de 1982 à 1983, puis implanté en 1985. Désormais installee dans deux ordinateurs VAX, la banque $d u$ Secrétariat d'État allait tourner sur le logiciel BASIS. Ayant récupéré la technologie Télidon, elle pourra contenir des graphiques en couleurs et mettra à la disposition de l'utilisateur la consultation par menus, l'affichage des données terminologiques en continu ou en parallèle, la personnalisation du système grâce au profil d'usager, etc. (Adshead $1985: 63-64$ ).

\section{Ressources}

TERMIUM était une banque bilingue (anglaisfrançais) jusque-là. Avec TERMIUM 3, il devient possible d'y consigner des données en d'autres langues, soit pour l'essentiel de l'espagnol et de l'allemand. Mais la fiche de terminologie n'en est pas devenue multilingue pour autant: dans le fonds multilingue, la fiche bilingue contient de l'anglais (surtout) ou du français comme langue de départ et un équivalent dans la langue cible (Adshead $1985: 64-65$ ). Ce fonds multilingue contenait 33000 fiches en 1988 (Wells $1988: 58$ ) ; il fait partie de la base de données linguistiques, la principale, celle autour de laquelle sont organisées les trente-quatre autres bases de données du système. Parmi ces dernières, on trouve une base de données sources (ou documentaires) où sont décodées les mentions de sources qui apparaissent sur les fiches de la base de données linguistiques, tandis que les autres ont des fonctions de support ou de gestion.

La base de données linguistiques comporte quatre fonds: en plus du fonds multilingue déjà mentionné, elle compte un fonds terminologique, un fonds d'appellations (noms anglais et français de programmes, lois, organismes, etc., le plus souvent canadiens) et un fonds des difficultés de traduction où sont traités des problèmes de morphologie, faux amis, syntaxe, phraséologie, etc. (Adshead 1985 : 64 ; Célestin 1990 : 1 ; Wells $1988: 58,64)$. Le total d'un million de fiches (pour les quatre fonds) a été dépassé en janvier 1991.

En livrant TERMIUM au Secrétariat d'État, l'Université de Montréal lui cédait du même coup un système de classement terminologique (Adshead $1985: 67$; Andersen $1982: 10$ ).

La classification par domaine des fiches terminologiques dans TERMTUM [...] se fait selon une structure hierarchique à trois niveaux et à cadre alphabétique. Vingt-trois classes constituent l'armature du classement et regroupent les domaines fondamentaux d'activité qui se prêtent à un développement terminologique. Chacune de ces classes comporte jusqu'à 26 divisions qui représentent à l'intérieur de la classe les principales aires d'activité, et les divisions se divisent à leur tour en un maximum de 26 sections représentant autant de situations terminologiques caractérisées par un centre d'activité. (Leonhardt $1990: 81$ )

Le thésaurus de TERMIUM a un rôle de classement et de délimitation de l'aire d'usage des termes. Les domaines qui en font partie recouvrent un réseau notionnel. Le dictionnaire des domaines où est identifié, situé et défini chacun des échelons du thésaurus constitue justement l'une des trente-cinq bases de données. On peut inscrire un maximum de trois domaines sur chacune des fiches de terminologie: quand un rédacteur le fait, il les place par ordre d'importance. En consultation interactive, les descripteurs de domaine d'emploi apparaissent en clair, alors que dans la base de données thésaurus ils prennent la forme d'un code. Les descripteurs (la désignation des domaines ou, pour être plus précis, leur code) sont enfin utilisés quand la Direction de la terminologie doit traiter des lots de fiches pour extraction, mise à jour, publication, etc. (Leonhardt 1990 : 81-85).

\section{Exploitation}

En janvier 1976, un total d'environ quatre-vingts terminologues travaillaient à la Division de la recherche terminologique et linguistique (Kerpan 1977: 51). Quelques années plus tard, Rondeau (1984: 168) évaluait le personnel de la DT à 200 employés, terminologues, personnel de gestion, soutien administratif, etc. Cardin (1984a : 18), parlant d'un situation postérieure, avance quant à lui le chiffre de 150 employés, dont une centaine en terminologie et une cinquantaine affectés à des tâches de gestion, promotion, techniciens et professionnels confondus. Il convient enfin de noter que les terminologues du Bureau des traductions ont d'autres tâches que l'entretien des fonds terminologiques de la banque.

Dans TERMIUM 3, en 1985, les mises à jour, enregistrées quotidiennement, touchaient environ 2200 fiches par semaine (Adshead 1985: 67; Leonhardt 1990: 86). On en est maintenant (Célestin $1990: 3)$ à 5000 mises à jour mensuelles : ce nombre représente le total de fiches touchées par des ajouts, des modifications, des suppressions, etc. Il existe aussi un programme qui dépiste automatiquement et rejette les doublets; on ne l'applique pas à l'ensemble de la base de données linguistiques mais seulement à des lots de fiches, à un sous-domaine, par exemple. L'orientation des recherches effectuées par la Direction de la terminologie est surtout fixée par le Bureau des traductions, quoique les demandes de mise à jour signalées par courrier électronique (intégré à la banque), en provenance du réseau de diffusion donc, ont aussi quelque influence.

L'information terminologique est consignée sur une fiche uninotionnelle, le plus souvent bilingue, 
dont on avait conçu le modèle au moment de TERMIUM 1 (Larose 1976: 3) et qu'on a revue avec la nouvelle génération. Toutes les données y apparaissent en clair sauf les sources des informations terminologiques qui, elles, ont été codées et qu'on déchiffre en consultant la base sources (Adshead 1985: 65-67). L'alimentation de la base de données linguistiques puise à de nombreuses sources: on y trouve des fiches de traduction épurées, de nouvelles fiches produites à la suite de recherches thématiques et ponctuelles menées au Bureau des traductions, des données obtenues auprès d'autres organismes, le dépouillement d'ouvrages et de textes divers, etc. (Larose 1976: 3). On comprend alors que les fiches possèdent un degré variable de fiabilité, qu'elles sont plus ou moins complètes, que l'information y est quelquefois très juste et quelquefois approximative. Aussi la mise à jour et le traitement de la redondance y constituent-ils des préoccupations importantes (Adshead 1985: 67; Cardin 1984a : 18; Cardin 1984b : 206). Même si on envisage depuis le milieu des années 1980 de procéder à une alimentation systématique et massive par l'acquisition de collections terminologiques produites par d'autres organismes, le problème du vieillissement fait maintenant son apparition et on decouvre que bon nombre de fiches antérieures à 1980 sont déjà périmées (Célestin 1990 : 3). Enfin, à choisir entre l'ajout de termes absents et l'ajout de définitions sur des fiches lexicales, la Direction de la terminologie penche actuellement en faveur de la première solution.

D'abord conçue pour répondre aux besoins des traducteurs, TERMIUM (dans sa version actuelle, c'est-à-dire TERMIUM 3) privilégie la recherche immédiate pour répondre à des questions ponctuelles ; l'accès direct y est donc considéré comme le mode de diffusion par excellence. Chaque usager intégré au réseau de diffusion peut personnaliser son accès à la banque : il gère son propre mot de passe, il détermine le mode et le format d'exposition, il choisit dans quelle langue, français ou anglais, il conversera avec l'ordinateur, etc. (Adshead 1985: 65; BT 1986). L'interrogation se fait au moyen de menus; on peut interroger terme par terme (mode dialogual) ou saisir une liste de termes (mode par lots). En mode dialogual et en cas de recherche positive, le logiciel produit un bilan par domaines, c'est-à-dire qu'il énumère dans une présentation plus ou moins élaborée selon le nombre de fiches repérées dans quels domaines elles sont classées. Parmi les autres possibilités qu'offre le système, il convient de mentionner la consultation des index alphabétiques français et anglais, l'impression de fiches en différé et la décomposition automatique des multitermes (Adshead 1985: 65; BT 1986). Un service de courrier électronique intégré permet à chaque utilisateur de communiquer avec la direction de la banque et avec les autres abonnés. Un utilisateur peut ainsi signaler une recherche infructueuse ou une coquille qu'il a relevée, enregistrer une demande de mise à jour, etc. Enfin, l'accès à la banque est gratuit, l'usager n'ayant qu'à fournir les moyens matériels indispensables au branchement (micro-ordinateur ou terminal, logiciel de télécommunication, modem, etc.).

En différe, TERMIUM est surtout connue par le biais d'un service de consultation ponctuelle, le SVP, qu'on peut rejoindre par téléphone ou courrier. Le Bureau des traductions compte aussi sur un service d'édition actif qui publie des recueils lexico-terminographiques avec régularité.

À la suite d'une proposition soumise par une entreprise privée en 1985, le Secrétariat d'État fit le projet de transférer TERMIUM sur CD-ROM (pour Compact Disc Read Only Memory, ou Disque Optique Compact, $D O C$ en français) (Carey, Dupont et Leonhardt 1991 : 174). Les avantages que représentait un pareil virage technologique étaient nombreux, d'autant que le Secrétariat d'État prévoyait éprouver des difficultés avec l'accès direct à son système malgré l'introduction de TERMIUM 3 : le nombre d'utilisateurs en simultanée est en effet limité, la dispersion géographique des utilisateurs (six fuseaux horaires au Canada seulement) pose des problèmes de disponibilité horaire et la puissance des ordinateurs VAX dont dispose le Secrétariat d'État possède des limites qu'on a vite atteintes (Wells 1988: 59). Par opposition, le DOC reste disponible sans mot de passe, sans avoir à rallier un réseau de télécommunications, en tout temps. Il occasionne toutefois les déboursés nécessaires à l'acquisition du DOC et du lecteur de DOC (Carey, Dupont et Leonhardt 1991 : 179).

La première étape du projet pilote est lancée à l'automne 1987, puis à la suite des résultats qu'on obtient alors, on passe à la deuxième étape: des copies sont mises en circulation à l'intérieur du Bureau des traductions, dans les services de traduction d'entreprises privées et dans quelques universités afin de recueillir des commentaires. En version DOC, la fiche terminologique de TERMIUM a conservé seulement les champs utiles à la diffusion de l'information; la plupart des champs de gestion ont été éliminés. En consultation, les fiches se présentent en colonnes, l'anglais à gauche, le français à droite, et le format d'affichage implicite est l'affichage complet; il demeure cependant possible de créer d'autres formats selon les besoins de l'utilisateur. 915000 fiches occupent les deux tiers de l'espace mémoire du disque, tandis que les mots clés des entrées forment deux index, un anglais et un français, dans lesquels ni les mots-outils ni les signes de ponctuation ne trouvent place (Carey, Dupont et Leonhardt 1991 : 175-178).

Le logiciel d'interrogation fait appel à la technologie des fenêtres: l'écran se divise en trois secteurs bien délimités, l'un est réservé au choix des index, un autre à la formulation de la question et le troisième au déroulement de l'index choisi. Pendant la frappe du terme recherché, l'index est balayé. On peut ainsi choisir de consulter une fiche en pointant l'entrée correspondante dans l'index ou en commandant l'affichage du résultat positif de la recherche. L'emploi de la banque est enfin facilité par un ensemble de moyens comme le recours aux touches de fonction, des menus déroulants et des fenêtres de dialogue (Carey, Dupont 
et Leonhardt 1991 : 177). Après la mise en marché du système complet (c'est-à-dire doté d'extraits de la base de données sources) sur DOC, le Bureau des traductions envisage maintenant d'offrir une base de données multilingue, de rendre possible son utilisation en mode résident, de développer son interrogation en hypertexte et, enfin, de l'adapter au Macintosh. En 1990, on évaluait que le prix de vente devrait varier de 1100 à 1500 dollars par année, ce qui comprendrait trois mises à jour annuelles, la documentation afférente et la formation (Carey, Dupont et Leonhardt 1991 : 178-179; Célestin 1990: 4).

\section{Réseau}

La très grande majorité des utilisateurs de TERMIUM sont des traducteurs: ils ont véritablement façonné la banque à leurs besoins. Mais on aimerait qu'elle suscite le même intérêt auprès des rédacteurs, des clientèles spécialisées et même auprès des citoyens canadiens. Après tout, TERMIUM est une banque à vocation universelle et on souhaiterait en avoir fait une banque d'information (Cardin 1984b : 206). S'il lui est impossible de se détacher des traducteurs, de se dégager des entreprises et des organismes producteurs ou consommateurs de terminologie, sa direction apprécierait qu'elle puisse au moins se rapprocher de la population (Cardin 1984b : 209). De manière plus réaliste, les services du Bureau des traductions sont offerts aux 260000 fonctionnaires des ministères et organismes du gouvernement fédéral. Quant aux fonctions de recherche terminologique et de conseil linguistique, elles visent autant l'entreprise privée que le grand public (Wells 1988: 57). Malgré tout, en ce qui concerne TERMIUM, le rôle de promouvoir le bon usage des langues officielles au Canada reste encore à être développé (Wells 1988: 58-59).

Dans sa croissance, le réseau de diffusion de la banque a suivi les bornes du milieu de la traduction et de la terminologie. Dès l'acquisition et la refonte du système par le Secrétariat d'État, on crut que la meilleure méthode consistait en l'implantation de terminaux. Les succursales du Bureau des traductions, les services de traduction de l'administration fédérale et ceux des sociétés d'État se virent ainsi attribuer des terminaux à imprimante. C'est pourquoi, localement, on a longtemps parlé d'implantation des banques de terminologie en termes de terminaux. En 1979, $89 \%$ des traducteurs du Bureau des traductions avaient accès à un terminal; des soixante-deux terminaux installés, trois l'avaient été à l'extérieur du gouvernement (Rondeau 1984: 167). Ces chiffres ont progressé régulièrement au cours des années, de telle sorte qu'en 1988 (Wells 1988: 58-59), le réseau de TERMIUM comptait 288 terminaux dont vingt-sept à l'étranger, aux Etats-Unis et en Europe occidentale essentiellement (dans des organismes internationaux et des ambassades du Canada). Au Canada, la plupart des terminaux sont placés dans les locaux du Bureau des traductions à Ottawa, Montréal, Toronto, etc., ou dans les services de traduction de l'administration publique fédérale.

\section{La Banque de terminologie du Québec \\ Situation}

La Banque de terminologie du Québec (BTQ) est créée avec le mandat de mettre les ressources terminologiques du monde francophone à la disposition des Québécois afin de rendre possible la généralisation du français et afin d'assurer son maintien (Fortin 1985: 2). Ce mandat est réparti sur des objectifs qui ont pu être modifiés en surface selon les circonstances ou les ressources disponibles, mais qui sont fondamentalement restés les mêmes :

1) dresser un inventaire des terminologies répertoriées et des travaux de recherche terminologique en cours, tant au Québec qu'à l'échelle de la francophonie :

2) rassembler, centraliser et emmagasiner la terminologie nécessaire à la francisation du Québec;

3) mettre sur pied un réseau de production et de diffusion des terminologies produites et enregistrées en mémoire d'ordinateur ;

4) gérer ou coordonner les travaux terminologiques en sol québécois ;

5) foumir une assistance logistique et linguistique aux projets de recherche terminologique;

6) joindre le cercle international des organismes de terminologie en participant aux colloques, congrès, rencontres diverses, etc. ;

7) effectuer des recherches linguistiques, terminologiques, documentaires ou théoriques en matière de traitement automatique des données terminologiques pour satisfaire les objectifs de l'Office (Auger et al. 1973: 7 ; Fortin 1978a : 1-4; Fortin 1978b : 1-7; Fortin 1985: 2-3; Motard 1982: 26 ; Rondeau 1981: 157; Salvail 1976: 176-177).

Les limites du classement alphabétique avaient déjà été perçues en terminologie. On observait aussi que le support papier se périmait rapidement, tandis que, en comparaison, les avantages offerts par l'informatique augmentaient avec le temps (Fortin 1978b: 4). Dès 1969, l'OLF allait expérimenter un fichier semi-automatisé (Motard 1982: 25), mais ce dernier s'étant tévélé insuffisant, on amorce en mars 1973 les études préalables à la constitution d'un instrument souple et efficace, d'un véritable système intégré de production et de gestion des terminologies (Fortin 1978a : 1-4 ; Fortin 1988: 52; Motard 1982: 25). En décembre de la même année, l'OLF, en collaboration avec le Service de l'informatique du ministère de l'Éducation, amorce la transmission des données déjà accumulées dans son premier fichier informatise (Motard 1982: 25 : Rondeau 1981: 156). C'est ainsi que commence véritablement à se construire cet immense dictionnaire informatisé anglais-français doublé $d$ 'un inventaire permanent des ouvrages terminologiques élaborés dans toute la francophonie (Hébert 1986: 12).

On exprime souvent le vœu, à cette époque, qu'une banque de terminologie permette l'intégration de toutes les formes d'activités terminologiques au 
Québec. Ainsi, la BTQ deviendrait le dépôt aù chacun investirait les résultats de son travail et de ses recherches et, du même coup, elle nécessiterait la participation de tout le milieu de la traduction et de la terminologie (Salvail 1976: 177-179). La banque a par ailleurs été conçue à la suite d'une série de colloques sur les méthodes de travail en terminologie, sur le traitement informatique des données terminologiques, sur le contenu d'une fiche normalisée, etc.; ses concepteurs, employés de l'OLF, tenaient à ce qu'elle vive en symbiose avec l'appareil administratif de l'OLF et que les recherches terminologiques entreprises à l'OLF soient un produit assimilable par la BTQ (Auger et al. 1973: 7 ; Auger, Rousseau et coll. 1978: 11; Fortin $1984: 202$ ).

La première phase de développement de la BTQ consistait en un inventaire le plus complet possible des terminologies enregistrées et des travaux terminologiques contenant du français, au Québec d'abord et dans toute la francophonie ensuite (Fortin 1984: 202 ; Globensky 1983: 122). On allait ensuite passer au stockage partiel ou intégral des informations repérés, puis dans un troisième temps à l'analyse des données stockées (Globensky 1983: 112).

\section{Ressources}

Des circonstances évoquées jusqu'à présent s'ensuivront les structures qu'on donnera au système de la BTQ. Les données enregistrées seront réparties dans six bases de données constituant la banque: quatre bases seulement (INVENTAIRE, SOURCES, DIFFUSION et TRAVAIL) seront interrogeables en direct, tandis que les deux dernières (THESAURUS et ORBE) ne seront accessibles qu'en différé.

Le grand intêrêt porté aux sciences de l'information par les concepteurs de la BTQ n'est probablement pas étranger au choix du SGBD STAIRS d'IBM, celui qu'on utilise encore aujourd'hui. L'OLF a confié la gestion informatique de sa banque au serveur IST (Montréal) où on exploite des ordinateurs AMDAHL. La capacité totale de la base de traitement, répartie sur trois disques IBM 3350, est de 950 millions de caractères. STAIRS effectue la gestion des données, l'interrogation des bases (grâce à l'interface AQUARIUS), le chargement, le contrôle de sécurité, la production de statistiques, etc. Des travaux auxiliaires sont enfin exécutés sur un mini-ordinateur HP 3000 situé dans les bureaux de l'OLF à Québec (De Bessé 1983 : 163; Hotz 1983 : 58; Rondeau 1981: 158).

La base INVENTAIRE, ainsi désignée parce qu'elle contient l'inventaire des ouvrages, travaux et recherches terminologiques comportant du français, fut la première à être mise sur pied et à être exploitée. Elle se présente comme une base de données documentaires où les informations (titre de l'ouvrage, auteur, vedette-matière, un ou des lieux de consultation, etc.) sont enregistrées sur une fiche bibliographique. Appelée TERMINOQ II pendant des années, elle informe des ouvrages, publications, chantiers et autres qui représentent un intérêt ou une dimension terminologiques. À l'OLF même, la démarche de recherche terminologique voulait, dès 1978, qu'on entreprenne tout chantier par l'établissement d'un état de la terminologie propre à un domaine en consultant la base INVENTAIRE. C'est qu'elle ne contient pas que des références d'ouvrages terminologiques, mais aussi celles d'ouvrages paraterminologiques comme des catalogues, des monographies, des normes techniques, des modes d'emploi, etc. On y dénombrait 25000 fiches en 1982, 30000 en 1983, 38000 vers 1986-1987, alors qu'à la fin mai 1991 on atteignait un total de 45878 références. On l'enrichissait au rythme approximatif de 2000 titres par année en 1987. (Auger, Rousseau et coll. 1978: 16; De Bessé 1983: 163; Gagnon et Hébert 1988: 18-22; Hébert 1986: 12; Hébert 1988: 58; Hotz 1983 : 58: Marchand 1976b : 219 ; Motard 1982 : 27)

Quant aux terminologies elles-mêmes, du moins celles qu'on considère nécessaires à l'OLF et à l'entreprise, elles sont consignées dans deux bases de données, DIFFUSION et TRAVAIL, qu'on désigna longtemps par TERMINOQ I, interrogeables en simultanée ou séparément. Les données sont enregistrées sur une fiche de terminologie divisée en soixante-huit champs, chacun étant réservé à un type d'information particulier. Parce que destinée à la francisation d'un Québec noyé dans une mer d'anglophones, la fiche BTQ, uninotionnelle, atteste des usages anglais et français; il suffit donc qu'elle soit bilingue. Dans les faits, en 1983 (Globensky 1983: 114), les fichiers terminologiques de la BTQ comportaient $85 \%$ de fiches bilingues, le reste étant unilingue (anglais ou français); et du total des fiches bilingues, $40 \%$ comportaient aussi des informations terminologiques complémentaires comme des définitions, contextes ou notes. Après un certain nombre de champs de gestion (numéro séquentiel, sigle de rédacteur, sigle d'organisme, date de rédaction, domaines d'emploi, etc.), la fiche BTQ comporte une partie anglaise (entrées principale et secondaires, contextes, définitions ou notes), une partie française et les codes de sources correspondant aux reférences d'origine des informations qui composent la fiche.

Même si on les consulte en simultanée dans la très grande majorité des cas (sous l'appellation CONC, pour concaténées), les bases de données DIFFUSION et TRAVAIL se distinguent par le type d'information qu'elles contiennent et par leur mode d'intégration à la chaine de production terminologique propre à la BTQ. On a longtemps réservé la base DIFFUSION aux fiches produites par des organismes nationaux ou internationaux de normalisation comme la CEI (Commission Électrotechnique Internationale), Ia CTOLF (Commission de terminologie de l'OLF) l'AFNOR (Association française de normalisation), l'ONGC (Office des normes du gouvernement canadien), etc. À la suite de l'épuration des données terminologiques, on y a classé des fiches dont le contenu présentait un degré de fiabilité élevé en termes d'équivalence et d'intégrité notionnelle. Fin mai 1991, DIFFUSION contenait 367640 fiches. Quant au fichier TRAVAIL (contenu à la fin mai 1991: 486 
251 fiches), on y a surtout consigné des fiches bilingues de type lexical, occasionnellement des fiches unilingues où le terme est accompagné d'informations définitoires ou contextuelles, ou encore des données qui ont une valeur descriptive ou informative au sujet de la notion traitée. Mais depuis la création d'un fichier PISTE (contenu à la fin mai 1991: 1427 fiches) en 1988 , on y retrouve de plus en plus de fiches étoffées, bien documentêes; ce dernier fichier est destiné à l'enregistrement de données fragmentaires ou dont l'équivalence des termes anglais et français paraît incertaine. Fin mai 1991, le contenu total des fichiers terminologiques de la BTQ s'élevait à 855318 fiches (BTQ 1991; Fortin 1978a: 4; Gagnon et Hébert 1988: 8-13; Globensky 1983 : 114; Hébert 1986: 12; Hébert 1988 : 57-58; Hotz 1983 : 58; Marchand $1976 \mathrm{~b}: 224$; Rondeau $1981: 158$ ).

La quatrième base de données accessible en direct, SOURCES, contient les références des sources d'information consultées pour rédiger les fiches de terminologie des bases de donnees terminologiques, qu'il s'agisse d'ouvrages lexico-terminographiques, monographiques, encyclopédiques, de personnes, de comités, de périodiques, etc. Chacune des informations terminologiques consignée sur une fiche étant identifiée par un code, celui-ci sert de lien entre les deux bases de données. On y dénombrait 27900 références fin mai 1991, soit le nombre total de sources consultées pour la constitution des bases de données terminologiques de la BTQ (De Bessé 1983: 163; BTQ 1991; Gagnon et Hébert 1988: 13-17; Motard $1982: 26-27$ ).

Le nom du premier des deux fichiers non accessibles en direct, ORBE, se décode Organismes, Rédacteurs, Bibliotheques, Entreprises : on y a répertorié le décodage en clair de tous les sigles utilisés sur les fiches documentaires ou terminologique de la Banque. La désignation Organisme renvoie aux sigles d'organismes apparaissant sur les fiches (de terminologie ou documentaires), Rédacteur au code particulier de chaque rédacteur qui a produit des fiches aussi bien documentaires que terminologiques, Bibliothèque aux sigles apparaissant dans le champ «lieu de consultation" des fiches documentaires, Entreprise aux sigles apparaissant surtout sur les fiches de terminologie fournies par les entreprises, qu'elles soient ou non abonnées à la BTQ. (De Bessé 1983: 164 ; Motard 1982 : 26-27)

Quant au thésaurus des domaines d'emploi, objet de la sixième et dernière base de données, il

est le plan de classement des fiches de terminologie. [...] constitué de l'ensemble des domaines d'emploi et des relations entre les domaines d'emploi et des relations entre les domaines qui structurent ce plan de classement, il est organisé sous la forme d'arbres qui en donnent la structure hiérarchique. (Trudel 1990: 131)

Un tel dispositif de classement sert d'abord a regrouper les fiches terminologiques enregistrées dans la Banque, à les indexer et, ainsi, à orienter le repérage des terminologies par domaine. Quant aux arbres, ils sont la représentation hiérarchique, plus ou moins arbitraire, de l'organisation des connaissances dans tel ou tel domaine d'activités, comme le déterminent les besoins des langagiers à certains moments clés de l'histoire des sciences et des techniques. Le thésaurus de la BTQ compte 106 domaines divisés en arbre et 104 autres qui ne le sont pas; le nombre de fiches classées dans ces derniers ne justifiait pas l'élaboration d'un arbre. Lors de la mise en chantier terminologique d'un domaine particulier, on vérifie sa représentation dans le thêsaurus. Quand il en existe un déjà, on complète ou on corrige l'arbre; s'il n'en existe pas, on en produit un (Auger, Rousseau et coll. 1978: 16-9; De Bessé 1983 : 164 ; Globensky 1983 : 116; Hotz 1983: 60; Motard 1982: 26-27; Trudel $1990: 131-137)$

Les ressources en personnel affectées à la BTQ varient selon les aléas politico-économiques de l'administration publique; elles sont d'ailleurs aussi difficiles à évaluer que pour les autres banques : si un certain nombre d'employés travaillent directement à l'alimentation ou à l'entretien des fichiers de la Banque, d'autres y occupent des fonctions marginales, et l'existence de leur emploi demeure tout à fait hypothétique advenant la disparition de la BTQ. Pour sa part, Nada Kerpan (1977: 52) évaluait à 150 le nombre de terminologues à l'emploi de la Direction de la terminologie en 1977. Or, à l'époque, la BTQ formait un des trois services de cette direction (Marchand 1976b: 219) et ne pouvait accaparer qu'une fraction de ce total. La constitution de la BTQ fut par ailleurs envisagée comme une entreprise conjointe, le principe de division des tâches assignant aux employés de l'OLF la responsabilité des recherches terminologiques dans les trones communs industriels et de gestion, tandis que les langagiers des entreprises assumeraient les recherches dans les vocabulaires spécialisés (Salvail 1976: 179). Mais il paraît difficile de calculer combien d'années-personnes les contributions du secteur privé ont représenté au cours des années, ou même si elles ont produit, sauf exception, un effort le moindrement appréciable... Quoi qu'il en soit, la structure actuelle de l'OLF concentre dans une direction unique, la Direction des services linguistiques (DSL), l'essentiel du travail de recherche terminologique. Et comme la BTQ représente le principe d'intégration de ces recherches, on peut plus ou moins considérer ces employés comme ceux de la BTQ : ils étaient quatre-vingt-quinze en avril 1991, dont près de quarante-cinq professionnels et techniciens directement affectés à l'alimentation et à la mise à jour des fichiers, la différence étant composée du personnel cadre, du soutien administratif, des personnes préposées à la gestion et à la diffusion. Pour avoir une idée précise des ressources nécessaires au fonctionnement de la BTQ, il faudrait enfin pouvoir quantifier le nombre d'employés du Service informatique de l'OLF et du serveur informatique (IST) qui traitent plus spécifiquement des dossiers en rapport avec la 
BTQ. Mais de telles données ne sont ni publiques ni même disponibles.

\section{Exploitation}

Comme on évaluait, au moment de la fondation de la BTQ, avoir besoin d'un minimum de 500000 termes pour offrir un taux de réponse convenable, on mit sur pied un programme de stockage massif au terme duquel on devait avoir emmagasiné 2100000 fiches dans le fichier TRAV, 700000 fiches dans le fichier DIFF et $\mathbf{5 0} 000$ fiches documentaires. En réalité, on disposait de seulement 20000 fiches corrigées et prêtes à être diffusées, tandis que la cadence de correction atteignait des crêtes de 6 à 7000 fiches par mois en 1976. En 1978, on emmagasinait environ 1440 fiches par semaine et on prévoyait ainsi atteindre bientôt le seuil du million de fiches. On $y$ parvint presque vers 1981-1982. Mais cette première phase de stockage massif devait se terminer en 1981. Depuis, grâce aux travaux de pré-épuration et de mise à jour, ce nombre a diminué (Fortin 1978a: 4 ; Fortin 1978b : 8-9; Fortin 1984: 202 ; Fortin 1985: 2-5; Globensky 1983: 114; Marchand 1976b: 223; Rondeau 1981: 157; Salvail 1976: 179).

Des terminologies non commercialisées, les résultats des recherches thématiques ou ponctuelles menées à l'OLF, les fiches transmises par les abonnés de la banque, le produit d'échanges de bandes magnétiques avec d'autres banques ou avec des organismes internationaux de normalisation, etc., sont ainsi enregistrés dans les mémoires de la BTQ. En plus de miser sur les ressources de son personnel, la banque $s$ 'est constitué un réseau d'alimentation externe. Ce dernier se compose d'abord des producteurs de terminologie qu'on a tenté d'intégrer au processus de production terminologique dans la perspective plus large de la francisation du Québec: des comités interentreprises animés par des terminologues de l'OLF ont été créés en vue d'uniformiser la terminologie de certains secteurs; quant aux abonnés de la BTQ, ils ont été invités à troquer leur production terminologique contre du temps d'accès à la Banque ${ }^{5}$. Le réseau d'alimentation externe se compose enfin des organismes internationaux avec qui l'OLF entretient des rapports de coopération et d'un certain nombre d'universités, surtout québécoises, qui permettent à leurs étudiants de se familiariser avec la BTQ et avec les méthodes de travail de l'OLF' (Fortin 1985:3-5; Hébert 1988 : 58 ; Hotz 1983 : 58 ; Rondeau $1981: 158$ ).

L'alimentation massive des fichiers terminologiques telle que pratiquée jusqu'en 1981 ne fut pas sans soulever quelques problèmes: elle engendra d'abord un fort taux de redondance, puisqu'on versait intégralement le contenu d'un certain nombre d'ouvrages lexico-terminographiques dans chaque domaine et que ces demiers ont tendance à reprendre un noyau commun de notions. Autre problème consécutif au stockage massif, l'indexation inadéquate: souvent, tout le contenu d'un document était classé dans un domaine unique; or il arrive fréquemment que des notions appartenant à divers domaines se côtoient dans un ouvrage, même le meilleur... D'autre part, on pratiqua, jusqu'à l'abus parfois, l'indexation multiple, qui consiste en l'attribution de quelques domaines d'emploi différents à une notion. Enfin, l'effort nécessaire au traitement et à l'enregistrement de centaines de milliers de fiches terminologiques eut préséance sur la question épineuse des droits d'auteur. Jusqu'au tournant des années 1980, on avait négligé, tant à l'OLF que partout ailleurs, la réflexion qu'imposaient les nouveaux moyens de diffusion créés par les sciences de l'information.

On adopta donc diverses mesures pour corriger ces problèmes. Par exemple, on commença dès le début des annćes 1980 à s'interroger au sujet des droits d'auteur: on tira alors la conclusion qu'il convenait de rétribuer les auteurs de documents terminologiques stockés dans la BTQ et que la qualité des relations avec le milieu de la traduction et de la terminologie importait plus que les sommes d'argent en jeu. Pour régler les deux autres problèmes, on éprouve une solution unique: de gigantesques travaux de mises à jour par secteurs, dénommés pré-épuration sont entrepris en 1981. Ils sont destinés à éliminer la redondance, d'une part, et l'indexation multiple ou erronée, d'autre part. On croit alors qu'au terme de cette activité, la fiche de terminologie deviendra le support d'une information notionnelle unique par domaines. La pré-épuration aura comme conséquence d'accroître la taille du fichier DIFFUSION en produisant une information de plus grande qualité parce que validée, tandis que le fichier TRAVAIL, dont la vocation informative est préservée, diminuera dans les mêmes proportions. À moyen terme, l'analyse et le traitement sectoriels des terminologies visaient deux effets sur l'utilisation finale: 1) les domaines épurés pouvaient être consultés sans bruit, et 2 ) les synthèses auxquelles on aboutissait pouvaient donner lieu à la publication d'états terminologiques, publications de type lexical qui devaient rendre compte de l'état des fichiers à un moment précis de leur évolution. De 1981 à 1984,450000 fiches appartenant à trente-huit domaines importants furent ainsi touchées. Au cours de l'exercice 1984-1985, on prévoyait en traiter 200000 de plus. En 1986-1987, 152000 furent mises à jour ( 36200 fiches nouvelles et modification ou suppression de 115700 fiches), ce qui représentait alors $18 \%$ du contenu de la banque. Il est facile d'imaginer que le traitement de telles masses de fiches imposait des structures administratives adéquates ainsi que des réorganisations continuelles des effectifs, ce qui ne manqua pas d'être fait (Fortin 1984: 202203; Fortin 1988: 54 ; Globensky 1983: 114 117; Motard 1982: 28).

Les modes de diffusion du contenu de la BTQ ont évolué avec les années. La téléinformatique ne comptant que de très rares usagers vers la fin des années 1970 , les terminologies de la Banque furent d'abord diffusées en différé. Dès 1977, un service de consultation est mis sur pied à qui les usagers peuvent soumettre leurs problèmes linguistiques ou terminologiques, par téléphone ou par lettre. Même si les 
modalités du service ont changé, il existe encore une équipe de terminologues préposés aux consultations ponctuelles, à qui les usagers adressent quelques questions (cinq au maximum) pour obtenir une réponse rapide (soit immédiate, soit différée de quelques heures ou de quelques jours tout au plus). Toujours en différé, celui (individu ou entreprise) qui doit appliquer un programme de francisation peut transmettre par l'entremise de la Direction de la francisation des listes de termes de longueur variable. En pareil cas, le temps de réponse peut augmenter, surtout si certains des termes ne sont pas consignés dans la BTQ. On parle d'accès différé à la BTQ, parce que c'est un terminologue de l'OLF qui exécute la recherche.

Depuis 1980, l'usager peut devenir utilisateur en pratiquant l'accès direct : en se servant d'un terminal, d'une machine de traitement de texte ou d'un microordinateur, il se branche directement à la BTQ et y effectue lui-même ses recherches. Il doit cependant acquérir la maitrise d'un langage d'interrogation à peu près identique à celui des banques de données documentaires: on y retrouve des commandes, des opérateurs booléens, des qualificatifs de champ, des symboles divers comme la troncature, etc. (Fortin 1978a : 5 ; Fortin 1988: 52 ; Gagnon et Hébert 1988 : 23-39; Globensky 1983: 112-113 ; Hébert 1986 : 12 ; Hotz 1983 : 62 ; Marchand 1976b : 224 ; Motard 1982 : 27-29; Rondeau 1981: 158; Salvail 1976:219)

En plus des modes d'accès direct et différé, la BTQ diffuse son contenu sous forme imprimée. En fait de publications à caractère terminologique, on a produit des répertoires bilingues (anglais-français) et des états terminologiques. Comme publications à caractère documentaire, des données tirées des bases SOURCES et INVENTAIRE ont été réunies en bibliographies sélectives et diffusées de cette manière. La banque émet aussi, sur demande, des listes de termes informatisées ou des lots de fiches terminologiques. Enfin, pour échanger avec d'autres banques, le contenu de la BTQ est diffusé sous la forme de rubans magnétiques. D'autre part, les publications de l'OLF sont élaborées en très étroite collaboration avec la BTQ et donnent la plupart du temps un aperçu du contenu de la banque dans un sous-domaine particulier (Fortin 1978a : 5 ; Fortin 1988: 52 ; Globensky 1983: 112-113; Krommer-Benz 1985 : 108 ; Motard 1982 : 27-29; Rondeau $1981: 158$ ).

\section{Réseau}

À la différence des autres grandes banques, le réseau de diffusion de la BTQ ne peut s'appuyer sur un service de traduction important qui garantisse une demande considérable. Au départ, on s'interrogeait donc sur l'identité de la clientèle éventuelle d'une pareille banque (Salvail 1976: 179). Ce furent les circonstances politiques qui tranchèrent: la clientèle cible serait constituée des entreprises, organismes ou individus soumis aux dispositions de la Charte de la langue française. Mais l'accès direct n'étant pas encore disponible, la diffusion des ressources de la banque s'organisa d'abord suivant un axe interne : les publications et les services de diffusion en différé (consultation, traitement des listes de termes) mettraient à la disposition des usagers externes les terminologies colligées dans la banque et traitées par du personnel de l'OLF.

Le réseau de diffusion externe par accès direct s'élabore à partir de 1980. On y adhère par abonnement; ceux qui vont le composer proviennent de milieux assez divers: le milieu de la terminologie est le premier représenté, puis vont s'ajouter les services de traduction de grandes ou moyennes entreprises, des langagiers de l'administration publique, des traducteurs pigistes, des bibliothèques, des collèges et des universités, des organismes internationaux, etc. En 1982, la banque devient accessible sur DATAPAC, ce qui permet d'étendre le réseau d'abonnés à l'ensemble du territoire québécois, à l'Ontario, au Nouveau-Brunswick, à la Colombie-Britannique, à la France, aux États-Unis, etc., avec une forte concentration des abonnements à Montréal, Toronto, Québec et Ottawa. Jusqu'en 1990, on recueille ainsi près de 550 abonnements selon la formule de l'abonnement gratuit et de la facturation proportionnelle au temps d'utilisation. Au cours de cette période, environ 3000 utilisateurs sont formés à la consultation des fichiers. Puis la formule d'adhésion au réseau est modifiée: on s'abonne désormais en versant un montant forfaitaire de 200 dollars par année en échange d'une utilisation illimitée.

Le système est disponible de sept heures à minuit en semaine, et de neuf heures à dix-sept heures les samedi et dimanche afin d'accommoder le plus grand nombre d'utilisateurs. Les abonnés américains et européens doivent assumer leurs frais de télécommunications jusqu'au réseau DATAPAC. Enfin, l'acquisition, l'entretien et la modernisation du matériel informatique nécessaire à l'interrogation (micro-ordinateur, terminal, logiciel de télécommunications, modem, etc.) demeurent l'entière responsabilité de l'abonné.

Les temps d'utilisation pondérés selon les réseaux interne et externe fourniraient un élément d'analyse supplémentaire, mais pour la BTQ comme pour les autres banques, ces données doivent rester confidentielles (quand elles sont disponibles). Mentionnons toutefois à titre d'information approximative et vague que l'ensemble du réseau externe interroge la banque durant des milliers d'heures chaque année. En ce qui concerne le réseau interne, la donné temps d'utilisation n'offre aucun intérêt; il serait plus approprié de parler ici d'utilité, du fait qu'à l'interne, la BTQ est employée à la fois comme outil de gestion, d'organisation, de consultation et comme média pour diffuser les terminologies françaises. Ainsi, par exemple, en ce qui concerne le volet diffusion, les deux succursales (Québec et Montréal) du Service des consultations et les dix bureaux régionaux de l'OLF répondaient à 60000 demandes ponctuelles exprimées par des représentants d'environ 850 entreprises sous administration de la Charte de la langue française en 1984. En 1990-1991, les mêmes diffuseurs (bureaux régionaux et Service des consultations désormais 
unifié et localisé à Montréal) donnaient 80000 consultations. Or, les terminologues du Service des consultations sont en communication constante avec la BTQ et ils comptent sur elle pour répondre à une grande proportion des questions. Les temps d'utilisation cumulés (réseaux interne et externe) atteignent ainsi des milliers d'heures par mois.

Avec la BTQ, en somme, l'OLF a mis au point un média intégré qui informe autant des terminologies qui y sont enregistrées que des productions terminologiques importantes, des projets de publications, des chantiers, etc. S'il est vrai que la BTQ a profité, comme cadette du groupe, de l'expérience des autres grandes banques, elle a apporté un trait particulier, original, celui de se consacrer à la recension des ouvrages d'intérêt terminologique anciens ou récents et des travaux en cours. Cette vocation documentaire est peut-être attribuable, ou liée en tout cas, au fait que la banque a été créée avec le logiciel STAIRS, destiné au traitement des données documentaires et très populaire, à l'époque, dans le milieu des sciences de l'information et de la documentation.

Comme elle n'a pu s'appuyer sur un important service de traduction, qui l'aurait alimentée de ses recherches aussi bien que de ses besoins, elle a dû se créer rapidement un réseau de diffusion externe. Et ce trait aussi est particulier à la BTQ : la seule à ne pas disposer d'un large bassin interne de traducteurs pour justifier les fondements et les orientations de ses recherches, elle a dô chercher à l'extérieur une raison d'être, une utilité et des indices de son efficacité dans les activités traductionnelles. Enfin, en ce qui concerne sa constitution et les méthodes des recherches qu'on y poursuit, la BTQ a été strictement soumise aux nécessités et aux impératifs de la discipline terminologique, la traduction et les traducteurs n'étant pas intervenus dans son élaboration.

\section{CONCLUSION}

À part la BTQ, donc, les grandes banques ont basé leur développement sur les demandes issues d'un imposant service de traduction: la rentabilité immédiate d'un tel investissement devait passer, semble-t-il par l'augmentation de la productivité du service de traduction, par la réduction du temps consacré à la recherche terminologique à des fins de traduction et par la diminution du taux d'erreur dans les travaux de rédaction et de traduction techniques. Il faut bien voir cependant que dans certaines situations géolinguistiques particulières, des objectifs socio-culturels ou politiques se sont substitués aux impératifs du capital : le réseau des banques scandinaves (NORDTERM), la banque de la Generalitat de Catalunya (TERMCAT) la BTQ et d'autres encore n'existeraient pas autrement.

Comme il a été exposé, chacune des banques décrites satisfait aux exigences des critères identifiés dans la première partie.

1) Ressources financières: nul besoin d'examiner leur budget respectif pour constater que des millions de dollars ont été affectés, au long des années, aux postes budgétaires «salaires», «administration», «acquisition de terminologies», «achat de matériel», "contrats de service», etc.

2) Nombre de termes: les porte-parole des cinq grandes banques déclarent tous avoir traité plus de quelques millions d'entrées consignées sur un minimum d'un demi-million de fiches.

3) Ressources informatiques: elles suivent l'évolution de l'informatique (matériel et logiciel), passant de la grande informatique à la microinformatique.

4) Ressources humaines: bien qu'elles aient varié d'une banque à l'autre, d'une époque à l'autre, les ressources humaines engagées dans leur création et leur développement représentent des centaines, voire des milliers d'annéespersonnes; pas question en effet d'un terminologue seul ou encore de quelques traducteurs qui font de la terminologie à temps perdu...

5) Durée: même la plus jeune du groupe, la BTQ, atteint ses dix-neuf ans en 1992.

6) Réseau de diffusion: qu'il soit interne, externe ou mixte, chacune s'est constitué un réseau de diffusion particulier par lequel des terminologies d'entreprise, des vocabulaires choisis, des normes et recommandations sont directement acheminés aux utilisateurs cibles.

D'auxiliaires à la traduction (n'a-t-on pas déjà parlé de système de machine-aided translation), les banques de terminologie sont progressivement devenues des entités autonomes grâce, en grande partie, aux potentialites qu'offrait l'informatique : un fichier manuel ne servira jamais qu'à classer; en plus, un fichier informatisé permet la publication, la diffusion, la production de statistiques, la gestion des données, etc.

Les défis qui se posent aux grandes banques se sont transformés avec les contextes technique et socio-économique. À l'aube des années 1990, il est du plus grand intérêt d'observer comment, malgré les compressions budgétaires qui gangrènent les administrations occidentales, chacune négociera le virage de la domestication de l'informatique, contribuera à la croissance des industries de la langue, proposera des solutions aux problèmes actuels de la theorie terminologique.

On constate en tout état de cause que la multiplication des échanges internationaux et la mondialisation des marchés raffermissent l'avantage de leur position, à savoir que le canal linguistique est celui qu'on sollicite le plus dans les échanges de tous genres, comme en fait foi la croissance effrénée des besoins en traduction.

RENÉ GAGNON
Québec, Canada

\section{Notes}

1. Du nom de son concepteur, Hans Lenoch.

2. Logiciel de SGBD commercialisé par IBM. 
3. On dit aussi en différé.

4. On parle aussi d'accès direct ou mode conversationnel.

5. Cette formule est abolie depuis 1990

6. Sans être aboli, ce programme a été amputé des dispositions relatives à la production terminologique des étudiants, toujours en 1990.

\section{RÉFÉRENCES}

ABBOU, André et al. (1987): Les industries de la langue - Vol. 1: Applications industrielles $d u$ traitement de la langue par les machines, Paris, DAICADIF.

ADSHEAD, Mariam (1985) : «A New Look for TERMIUM ${ }^{2}$, TermNet News, $n^{\circ}$ 9, pp. 63-68.

ANDERSEN, Aldean (1982): «International Exchange Guidelines - applicable to Termium $\Pi$ ? $»$, L'actualité terminologique, 15-5, (mai 1982), pp. 8-10.

AUGER, Pierre et al. (1973): Guide de travail en terminologie, Québec, Office de la langue française, Cahiers de l'Office de la langue française, $\mathrm{n}^{\circ} 20$, $103 \mathrm{p}$.

AUGER, Pierre, Louis-Jean ROUSSEAU et coll. (1978): Méthodologie de la recherche terminologique, Québec, Éditeur officiel du Québec, Office de la langue française, $80 \mathrm{p}$.

BACHRACH, J. Albert (1971): «L'ordinateur au service du terminologue: maître ou esclave», Meta, 16-1/2 (mars-juin 1971), pp. 105-115.

BACHRACH, J. Albert (1981): «Historique d'un dictionnaire automatique», Terminologie Bulletin, $\mathrm{n}^{\circ}$ 38, pp. 25-29.

BACHRACH, J. Albert (1987) : «Une rétrospective et un regard sur l'avenir», Meta, 32-2 (juin 1987), pp. 97-101.

BAUDOT, Jean (1986): «Les banques de terminologie de l'avenir», Meta, 31-2 (juin 1986), pp. 153-158.

BENNETT, Paul A. et al. (1986) : Multilingual Aspects of Information Technology, Brookfield (Vermont), Centre for Computational Linguistics/Gower Publishing Co., $200 \mathrm{p}$

BERTELOOT, Pascale, MULDERS, Leo et Karsten WINTERHOFF (1988): «Translating and the Computer - Londres, 12-13 novembre 1987\%, compte rendu du congrès et en particulier de la communication de Wolfgang Nedobity, Terminologie et traduction, $\mathrm{n}^{\circ} 1, \mathrm{pp}$. 137-146.

BRINKMAN, Karl-Heinz (1981): «Machine Aids to Translation», Meta, 26-1 (mars 1981), pp. 67-75.

BT - Bureau des traductions (1986): Guide de l'usager TERMIUM, Ottawa, Direction générale de la terminologie et des services linguistiques, Secrétariat d'État du Canada, $37 \mathrm{p}$.

$B T Q$ - Banque de terminologie du Québec, [Accès direct], Québec et Montréal, Office de la langue française.

CARDIN, Michel (1983): «Les grandes banques de terminologie se concertent», L'Actualité terminologique, 16-7 (sept. 1983), pp. 8-9.
CARDIN, Michel (1984a): «La terminologie: un bond dans l'avenir», L'Actualité terminologique, 17-5/6 (juillet-août 1984), pp. 17-18.

CARDIN, Michel (1984b) : «TERMIUM - Les banques de terminologie et leur exploitation», ST, Proceedings. National Symposium on Linguistic Services, pp. 205-210.

CAREY, John, DUPONT, Sylvie et Christine LEONHARDT (1991): «TERMIUM sur CD-ROM», Meta, 36-1 (mars 1991), pp. 174-181.

CÉLESTIN, Tina (1990) : TERMIUM : Compte rendu de rencontres avec des gestionnaires du système, document photocopié, Québec, Office de la langue française, $4 \mathrm{p}$.

CIRB - Centre international de recherche sur le bilinguisme (1988): Les industries de la langue : au confluent de la linguistique et de l'informatique, Québec, CIRB, coll. «RELAI», ${ }^{\circ}$ K-9, $147 \mathrm{p}$.

CIRB - Centre international de recherche sur le bilinguisme (1989): La description des langues naturelles en vue d'applications linguistiques, Actes du colloque tenu à l'Université Laval du 7 au 9 décembre 1988, Québec, CIRB, coll. «RELAl», $n^{\circ} \mathrm{K}-10,344 \mathrm{p}$.

CLAS, André (1969) : «Éditorial : la banque de terminologie», Meta, 14-4 (décembre 1969), pp. 191194.

CLAS, André (1971) : «Éditorial», Meta, 16-1/2 (mars-juin 1971), pp. 5-6.

CLAS, André (1987): «Les nouveaux lexiques ou une stratégie de création de mini-banques», Meta, 32-2 (juin 1987), pp. 212-215.

COTY, Jean-Paul (1970): «L'actualité : Nominations à la Banque de terminologie», Meta, 15-3 (septembre 1970), pp. 195-196.

COUTURE, Bruno (1976): «La Banque de terminologie au service de l'entreprise», Meta, 21-1 (mars 1976), pp. 100-109.

DARBELNET, Jean (1965): «Pour une meilleure coordination de la recherche et de la documentation en terminologie», Culture, XXVI, pp. 328 330.

DE BESSÉ, Bruno (1983): «Réunion internationale des exploitants des banques de terminologie», $L a$ Banque des mots, $\mathrm{n}^{\circ} 26$, pp. 161-177.

DT - Direction de la terminologie (1982): La banque de terminologie de troisième génération, document photocopié, Ottawa, Secrêtariat d'État, août $1982,39 \mathrm{p}$.

DUBUC, Robert (1970): «L'actualitế : Colloque sur la langue française. Québec, 28 février et $1 \mathrm{er}$ mars 1970", Meta, 15-2 (juin 1970), pp. 137-140.

DUBUC, Robert (1972): «La Banque de terminologie de l'Université de Montréal», La Banque des mots, $\mathrm{n}^{\circ} 3$, pp. 35-50.

DUBUC, Robert et Jean-François GRÉGOIRE (1974): «Banque de terminologie et traduction», Babel, 20-4, pp. 180-184.

DUCHESNE, Carole (1982): Résultats de l'exercice d'identification des besoins des utilisateurs - 
Termium III, document photocopie, Ottawa, Secrétariat d'État, Direction de la terminologie, 24 février $1982,14 \mathrm{p}$. et annexes.

EURONET-DIANE (1987): DIANEGUIDE - Guide des bases et banques de données, Luxembourg, Euronet-DIANE, Groupe pour le développement du marché de l'information en Europe, $129 \mathrm{p}$.

FELBER, Helmut (1984) : Terminology Manual, Paris, UNESCO/Infoterm, $426 \mathrm{p}$.

FORTIN, Jean-Marie (1978a): La Banque de terminologie du Québec, un instrument d'information scientifique et technique de langue française, communication présentée au Colloque francoquébécois sur l'information scientifique et technique de langue française, Québec, avril 1978,6 p.

FORTIN, Jean-Marie (1978b): Le rôle et l' impact de la Banque de terminologie du Québec sur la francisation des entreprises, discours prononcé par Raymond Gosselin devant l'Association des manufacturiers canadiens, Québec, novembre 1978, $11 \mathrm{p}$.

FORTIN, Jean-Marie (1984): «La BTQ - Les banques de terminologie et leur exploitation», ST, Proceedings. National Symposium on Linguistic Services, pp. 201-205.

FORTIN, Jean-Marie (1985): La Banque de terminologie du Québec et la propriété intellectuelle, notes pour l'allocution présentée au $2^{e}$ Congrès annuel de la Société des traducteurs du Québec, document photocopié, Québec, juin 1985, 11 p.

FORTIN, Jean-Marie (1988) : «La Banque de terminologie du Québec : un instrument de développement social, au carrefour des enjeux technologiques et linguistiques», CIRB, Les industries de la langue : au confluent de la linguistique et de l'informatique, pp. 51-56.

FR.ANÇOIS, Pol (1981) : «La place de l'informatique dans EURODICAUTOM», Terminologie Bulletin, $\mathrm{n}^{\circ} 38$, pp. $155-158$.

GAGNON, René et Roselyne HÉBERT (1988): Guide d'interrogation de la Banque de terminologie du Québec, Montréal, Office de la langue française, 77 p., VII.

GLOBENSKY, Robert (1983) : «The Banque de Terminologie du Québec. An Instrument of Québec's Program to Work in French», Interrante et Heymann, Standardization of Technical Terminology: Principles and Practices, pp. 111-118.

GOBEIL, Fernand (1981): «La Banque de terminologie, un outil moderne au service des traducteurs», L'Actualité terminologique, 14-6 (juil. 1981), pp. 8-9.

GOETSCHALCKX, Jacques (1968) : «Langue et ordinateur», Babel, 14-1, pp. 49-51.

GOETSCHALCKX, Jacques (1981a) : «Eurodicautom: problèmes résolus et problèmes posés», Meta, 26-1 (mars 1981), pp. 76-80.

GOETSCHALCKX, Jacques (1981b): «Historique d'EURODICAUTOM», Terminologie Bulletin, $n^{\circ} 38$, pp. 31-34.
HÉBERT, Roselyne (1986) : «Un dictionnaire informatisé au service de la communication en français», Francisation en marche, 5,5 (octobre 1986), pp. 12.

HÉBERT, Roselyne (1988): «La Banque de terminologie du Québec», Kummer (Ed.), Across the Language Gap, pp. 57-59.

HIRSCHBERG, Lydia (1965) : «Dictionnaires automatiques pour traducteurs humains», Journal des traducteurs, 10-3, pp. 78-86.

HOHNHOLD, Ingo (1984): «The TEAM Terminology Data Bank System, Language Services Department, Siemens AG, Munich, Republic of Germany», TermNet News, $\mathbf{n}^{\circ} 8-1984$, pp. 19-33.

HOTZ, Adrien: Le repérage en terminologie informatisée, mémoire de maîtrise, Montréal, Université de Montréal, août 1983, $208 \mathrm{p}$.

HVALKOF, Sonja (1982): Étude comparative des données terminologiques des banques de terminologie DANTERM, B.T.Q., EURODICAUTOM, NORMATERM, OF.L. et Siemens, Arhüs, Danemark: École des hautes études commerciales et des langues modernes, 2 vol., 197 et $120 \mathrm{p}$.

ILJON, A. (1981) : «EURODICAUTOM - 15 Months on EURONET», Terminologie Bulletin, $\mathrm{n}^{\circ} 38$, pp. 151-153.

INTERRANTE, C. G. and F. J. HEYMANN (Eds.) (1983): Standardization of Technical Terminology: Principles and Practices. ASTM STP806, American Society for Testing and Materials, $146 \mathrm{p}$.

KERPAN, Nada (1977) : «Histoire de la terminologie au Canada et au Québec», Meta, 22-1 (mars 1977), pp. 45-53.

KROLLMANN, Friedrich (1971): «Linguistic Data Banks and the Technical Translator», Meta, 161/2 (mars-juin 1971), pp. 117-124.

KROMMER-BENZ, Magdalena (1985): World Guide to Terminological Activities: Organizations, Commissions, Terminology Banks, München, New York, London, Paris, K.G. Saur/Infoterm, Infoterm Series 4, $158 \mathrm{p}$.

KUMMER, Karl (Ed.) (1987) : Across the Language Gap - Proceedings of the 28th Annual Conference of the American Translators Association. October 8-11, 1987; Albuquerque, New Mexico, Medford (NJ), Leamed Information Inc. for the American Translators Association, $567 \mathrm{p}$.

LAROSE, Paul-Émile (1976): «Réorganisation des services terminologiques au Bureau des traductions - La banque de terminologie», L'Actualité terminologique, 9-2 (février 1976), pp. 1-4.

LEONHARDT, Christine (1990): «Le système de classement TERMIUM : exploitation et mise à jour», Revue québécoise de linguistique théorique et appliquée, 9-2 (mai 1990), pp. 81-106.

L'HOMME, Marie-Claude (1988): Origine et développement des industries de la langue, Québec, Centre international de recherche sur le bilinguisme, coll. «RELAI», publ. K-8, $147 \mathrm{p}$.

MARCHAND, Pierre (1976a) : «L'actualité : Conversation avec Marcel Paré, directeur de la Banque 
de terminologie de l'Université de Montréal», Meta, 21-2 (juin 1976), pp. 172-175.

MARCHAND, Pierre (1976b) : «L'actualité : Conversation avec Jean-Claude Corbeil, directeur de la terminologie à la Régie de la langue française», Meta, 21-3 (septembre 1976), pp. 219-225.

MENGARDUQUE, J. R. (1989) : «La terminologie au Service de traduction du Ministère néerlandais des Affaires êtrangères», Terminologie et traduction, $\mathrm{n}^{\circ} 3$, pp. 155-159.

MOTARD, Luce (1982): «La Banque de terminologie du Québec», TermNet News, $\mathrm{n}^{\circ} 6-1982$, pp. 25-29.

MOUZARD, François (1981): «La terminologie au gouvernement de la République fédérale d'Allemagne», L'Actualité terminologique, 14-3 (mars 1981), pp. 4-5.

QUICHERON, Jean-Bernard (1988) : «Congrès international sur la terminologie et le génie de la connaissance. Trèves, 29 sept.-1 ${ }^{\text {er }}$ oct. 1987», Terminologie et traduction, $\mathrm{n}^{\circ} 1-1988$, pp. 147-153.

REICHLING, Alain (1981) : «EURODICAUTOM II», Terminologie Bulletin, ${ }^{\circ} 38$, pp. 35-132.

RONDEAU, Guy (1981): Introduction à la terminologie, Montréal, Centre Éducatif et Culturel, $227 \mathrm{p}$.

RONDEAU, Guy (1984): Introduction à la terminologie, Chicoutimi et Québec, Gaëtan Morin éditeur, $238 \mathrm{p}$.

ROUSSEAU, Louis-Jean (1990) : «La pratique québecoise de la terminologie», Terminologies nouvelles, $n^{\circ} 3$ (juin 1990), pp. 68-71.

SAGER, Juan C. et R. L. JOHNSON (1979): «Terminology: The State of the Art», L'Actualité terminologique, 12-3 (mars 1979), pp. 1-3.

SAGER, Juan C. et John McNAUGHT (1980): Selective Survey of Existing Linguistic Data Banks in Europe, Manchester, Centre for Computational Linguistics, University of Manchester Institute of Science and Technology, dec. 1980, $80 \mathrm{p}$.

SALVAIL, Bernard (1976): «L'actualité : Compte rendu de la première rencontre des traducteurs et terminologues du Québec, tenue au Château Montebello, du 18 at 20 janvier 1976», Meta, 21-2 (juin 1976), pp. 175-179.

SCHNEIDER, Thomas (1987): «Terminotics of the Future : Three Scenarios», Meta, 32-2 (juin 1987), pp. 205-211.

SCHULZ, Joachim (1971): «Le système TEAM, une aide à la traduction», Meta, 16-1/2 (mars-juin 1971), pp. 125-131.

SCHULZ, Joachim (1980): «A Terminology Data Bank for Translators (TEAM)», Meta, 25-2 (juin 1980), pp. 211-229.

Secrétariat d'État, TERMIUM [Accès direct], Ottawa, Secrétariat d'État du Canada.

Secrétariat d'État (1991): TERMIUM [DOC], Ottawa, Secrétariat d'État du Canada.

SIEMENS AG (1989): Never at a Lass for Words. TERM-PC, the Terminology System, München, $\mathrm{K}$ Systeme AP., 4 p.
ST - Secretary of State (1984): Proceedings. National Symposium on Linguistic Services: Linguistic Services in Canada - Insight and Outlook. Actes du colloque national sur les services linguistiques - Ottawa du 9 au 12 octobre 1985. Bilan et prospective, Ottawa, Ministry of Supply and Services Canada, $409 \mathrm{p}$.

TRUDEL, Michèle (1990): «Hiérarchisation des domaines et mise à jour du système de classement de la Banque de terminologie du Québec», Revue québécoise de linguistique théorique et appliquée, 9-2 (mai 1990), pp. 131-148.

VOLLNHALS, Otto (1989): «TERM-PC - the Terminology System», TermNet News, $n^{\circ} 26$, pp. 20-23.

WELLENSTEIN, Hubert P. (1989): «Le système de classification Lenoch à l'usage des traducteurs, terminologues, documentalistes et bibliothécaires à la Commission des Communautés européennes», Revue québécoise de linguistique théorique et appliquée, 9-2 (mai 1990), pp. 107-111.

WELLS, Winston G. (1988) : «Termium : outil moderne de communication», CIRB, La description des langues naturelles en vue d'applications linguistiques, pp. 57-60. 